\title{
Onset of chaos in helical vortex breakdown at low Reynolds number
}

\author{
S. Pasche* and F. Avellan ${ }^{\dagger}$ \\ Laboratory for Hydraulic Machines, École Polytechnique Fédérale de Lausanne, 1007 Lausanne, Switzerland \\ F. Gallaire \\ Laboratory of Fluid Mechanics and Instabilities, École Polytechnique Fédérale de Lausanne, \\ 1015 Lausanne, Switzerland
}

(Received 6 November 2017; published 6 June 2018)

\begin{abstract}
The nonlinear dynamics of a swirling wake flow stemming from a Graboswksi-Berger vortex [Grabowski and Berger, J. Fluid Mech. 75, 525 (1976)] in a semi-infinite domain is addressed at low Reynolds numbers for a fixed swirl number $S=1.095$, defined as the ratio between the characteristic tangential velocity and the centerline axial velocity. In this system, only pure hydrodynamic instabilities develop and interact through the quadratic nonlinearities of the Navier-Stokes equations. Such interactions lead to the onset of chaos at a Reynolds value of $\mathrm{Re}=220$. This chaotic state is reached by following a Ruelle-TakensNewhouse scenario, which is initiated by a Hopf bifurcation (the spiral vortex breakdown) as the Reynolds number increases. At larger Reynolds value, a frequency synchronization regime appears followed by a chaotic state again. This scenario is corroborated by nonlinear time series analyses. Stability analysis around the time-average flow and temporal-azimuthal Fourier decomposition of the nonlinear flow distributions both identify successfully the developing vortices and provide deeper insight into the development of the flow patterns leading to this route to chaos. Three single-helical vortices are involved: the primary spiral associated with the spiral vortex breakdown, a downstream spiral, and a near-wake spiral. As the Reynolds number increases, the frequencies of these vortices become closer, increasing their interactions by nonlinearity to eventually generate a strong chaotic axisymmetric oscillation.
\end{abstract}

DOI: 10.1103/PhysRevFluids.3.064701

\section{INTRODUCTION}

Swirling flows experience vortex breakdown for a sufficiently large Reynolds number, as soon as the swirl intensity reaches a critical value. This bifurcation parameter is commonly associated with the swirl number $S$, defined as the ratio between the characteristic tangential velocity and the centerline axial velocity. Crossing this threshold, the columnar state of the vortex may bifurcate to several states characterized by a bubble, a spiral, or a double spiral breakdown configuration, which have all been reported in many experimental and numerical studies of recent decades (see Ref. [1] for a review). This sudden topological change impacts several industrial applications. It affects leading edge vortices that result in a poor flight performance [2] or it prohibits operating conditions in Francis turbines due to the development of large pressure fluctuations [3]. In contrast, the vortex breakdown may be also beneficial, especially in turbomachinery applications, to stabilize the flame of burners or to enhance the mixing of species [4].

\footnotetext{
*simon.pasche@alumni.epfl.ch

${ }^{\dagger}$ francois.avellan@epfl.ch

†francois.gallaire@epfl.ch
} 
Hydrodynamic instability theory applied to open flow has shed light on this phenomenon with the concept of absolute or convective spatially developing flows by Huerre and Monkewitz [5]. The presence of an advective-dominant direction on incoming fluctuations leads to two distinguished situations. In convectively unstable flows, unstable waves are swept away as they grow and the flow acts as a noise amplifier. In contrast, in absolutely unstable flows, unstable waves invade the entire domain withstanding the advection giving birth to a synchronized oscillator. This concept, applied to the Batchelor parallel vortex profile, has demonstrated that helical instabilities could become absolutely unstable [6]. Weakly nonparallel local stability analysis of the spiral vortex breakdown from the velocity profile of the three-dimensional (3D) direct numerical simulation (DNS) of Ruith et al. [7] has led to the interpretation of the spiral vortex breakdown as an absolute instability, triggered by the wake of the upstream bubble [8]. This interpretation was further validated by the global stability analysis of a nonparallel flow by solving the eigenvalue problem of the 3D linearized Navier-Stokes [9] equations around a base flow (see [10,11]). The vortex breakdown stemming from a Grabowski-Berger vortex in an open geometry has become a benchmark model for disturbance analysis (see $[12,13])$.

A bifurcation diagram of this benchmark model based on base flow investigations was reported by Meliga et al. [14], who highlight the bifurcation point where both single and double-helical modes become simultaneously globally unstable at the instability threshold. The selected mode is subsequently identified through a weakly nonlinear analysis for the bifurcation point $\operatorname{Re}=71.95$ and $S=1.436$. Nonlinear analyses of the vortex breakdown at low Reynolds number using numerical simulation focus on the formation of the bubble breakdown, the description of the flow patterns, and the validation of vortex breakdown criterion initially derived by Squire [15]. These DNSs were performed in several configurations such as in pipes by Lopez [16] or in closed containers by Escudier and Zehnder [17]. An early study that relaxed the radial confinement of the vortex breakdown was performed by Ruith et al. [7] with the aim to describe the linear stability of the first bifurcation state. Ruith et al. [7] have reported, in addition, the onset of a low frequency for larger swirl number $S=1.3$ and $\operatorname{Re}=200$ associated with a pulsant double-helical breakdown mode.

In contrast, studies investigating the nonlinear interactions of subsequent bifurcations were mainly performed in confined geometries. For example, spherical Couette flow [18] and Rayleigh-Bénard convection $[19,20]$ are the typical fluid flow systems presenting chaotic regimes. Turning back to swirling flow, Lagrangian chaos of vortex breakdown in closed containers was investigated by Sotiropoulos et al. [21] aiming at identifying the basin of attraction of particle trajectories to improve species mixing in swirl burner applications. In the same configuration, a quasiperiodic regime of Eulerian dynamics was reported by Serre and Bontoux [22] and Lopez [23] and Eulerian chaos was reported by Sørensen and Christensen [24].

Confined geometries exacerbate fluctuations of the bulk flow through a shear effect of the boundary layer and favor the system evolution to a chaotic regime acting as a consequence of reflection and feedback mechanisms, prior to leading to spatiotemporal chaos and turbulence. Chaotic regimes in open flows were also reported in bounded geometries, for instance, the converging-diverging channel flow of Amon et al. [25]. In the present study, the onset of Eulerian chaos in an open swirling wake flow inside a semi-infinite domain is investigated.

The route to chaos was found to follow three different possible universal scenarios [26-28]. The first one is the period doubling or Feigenbaum scenario where the consecutive period-doubling transitions are observed until chaos is reached. The second is the Manneville-Pomeau intermittency regime related to irregularly spaced bursts of chaos. The third scenario is the Ruelle-TakensNewhouse route where chaos is observed after the apparition of a strange attractor with three incommensurate frequencies. In this scenario, the successive bifurcations produced from a steady fixed-point solution to chaos proceed through the following states: First Hopf bifurcation yields a limit cycle, followed by periodic $k$-period oscillations when the secondary instability sets in with a commensurable frequency or alternatively a quasiperiodic state with two incommensurable frequencies, the torus $\mathbb{T}_{2}$. A subsequent bifurcation can yield a torus $\mathbb{T}_{3}$ with three incommensurable frequencies, which eventually becomes unstable and yields chaos. 


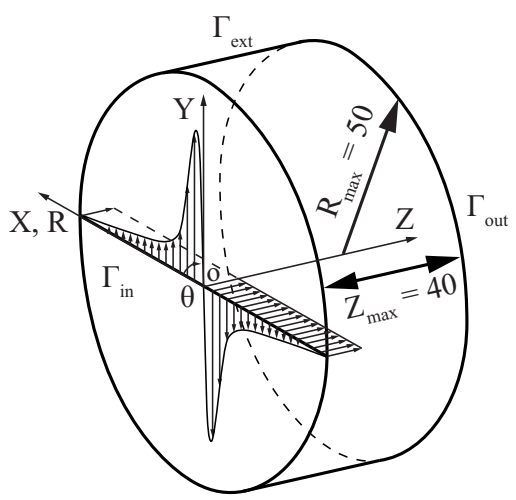

FIG. 1. Three-dimensional schematic of the flow configuration.

The route to chaos of the vortex breakdown phenomenon is investigated in an unconfined geometry where only pure hydrodynamic instabilities can develop. To this end, global stability analysis about the mean flow is considered, pushing the limit of such an analysis to identify multiple emerging modes and provide further insight into the route to chaos of the vortex breakdown.

The paper is organized as follows. The swirling wake flow of Ruith et al. [7] leading to the spiral vortex breakdown is described together with the flow configuration in Sec. II. A description of the numerical tools is presented in Sec. III, where the direct numerical flow simulation, the double temporal-azimuthal Fourier decomposition, and the global stability analysis are introduced to further study the emergence of flow patterns. Snapshots of flow vorticity isocontour for a selected set of parameters allow the reader to become familiar with the terminology used in the paper (Sec. IV) and a global preview of the flow dynamics is then presented as a bifurcation diagram in Sec. V. The system dynamics is investigated using nonlinear time series analysis in a single monitoring point (Sec. VI) and its sensitivity to initial disturbance (Sec. VIB) is shown to explicitly separate the chaotic from nonchaotic states, which we refer to throughout the rest of the paper. This local approach devoted to chaos theory and describing the flow state is then contrasted with the development of azimuthally periodic spatial modes computed by global stability analysis as well as through a double temporal-azimuthal Fourier series decomposition of DNS flow fields. These results are presented in Sec. VII. A summary is given and conclusions are drawn in Sec. VIII.

\section{FLOW CONFIGURATION}

We consider the dynamics of a Grabowsky-Berger [29] vortex, entering a semi-infinite domain $\Omega$ with the fluid assumed homogeneous and incompressible (see Fig. 1). The fluid motion can be described by the nondimensional incompressible Navier-Stokes equations made dimensionless by the characteristic vortex core radius and incoming centerline streamwise velocity

$$
\frac{\partial \mathbf{V}}{\partial t}+(\mathbf{V} \cdot \nabla) \mathbf{V}=-\nabla P+\operatorname{Re}^{-1} \nabla^{2} \mathbf{V} \text { in } \Omega, \quad \nabla \cdot \mathbf{V}=0 \text { in } \Omega,
$$

where $\mathbf{U}=(\mathbf{V}, P)$ is the state vector with $P$ the pressure, $\mathbf{V}=\left(V_{R}, V_{\theta}, V_{Z}\right)$ the velocity distribution based on standard cylindrical coordinates $(R, \theta, Z)$, and Re the Reynolds number of the problem built on the fluid's kinematic viscosity and the reference scales previously introduced. The dimensionless velocity profile of the Grabowsky-Berger [29] vortex prevailing at the inlet is written

$$
V_{R}(R)=0, \quad V_{\theta}(R)=S f(R), \quad V_{Z}(R)=1 \quad \text { on } \Gamma_{\text {in }},
$$


with $S$ the swirl number and $f(R)$ a piecewise function defined as

$$
f(R \leqslant 1)=R\left(2-R^{2}\right), \quad f(R>1)=1 / R .
$$

The dynamics of this vortex is investigated for a constant swirl number $S=1.095$, which restricts this study to the spiral vortex breakdown in contrast to pure bubble vortex breakdown or double-helix vortex breakdown observed at lower and larger swirl numbers, respectively [7,14]. Although this study focuses on spiral vortex breakdown, this system is observed to reach several dynamical states as the Reynolds number is increased. A bifurcation analysis based on direct numerical simulations (DNSs) is therefore performed up to $\mathrm{Re}=300$, using classical tools to investigate dynamical systems such as nonlinear time series, attractor cross sections, frequency identification, and sensitivity to initial disturbances. This approach, conducted at a single monitoring point, is then contrasted with the development of temporal and spatial 2D axisymmetric modes computed by global stability analysis around the mean flow and by temporal-azimuthal Fourier decomposition of the DNS.

\section{NUMERICAL METHODS}

\section{A. Direct numerical flow simulations}

Direct numerical flow simulations of the Navier-Stokes equations (1) are performed on a 3D cylindrical domain (Fig. 1). These equations are completed with the following boundary conditions: a Grabowsky-Berger vortex equation (2) at the inlet $\Gamma_{\text {in }}$, a free-outflow condition $\left[-P \mathbf{I}+\mathrm{Re}^{-1}\right.$. $(\nabla \mathbf{V})] \cdot \mathbf{n}=0$ on the external boundary $\Gamma_{\text {ext }}$, and a convective condition $\partial_{t} \mathbf{V}+\mathbf{U}_{c} \cdot \partial_{\mathbf{n}} \mathbf{V}=0$ at the outlet $\Gamma_{\text {out }}$, with $\mathbf{U}_{c}$ the convective velocity fixed equal to the free-stream velocity $\mathbf{U}_{c}=\mathbf{e}_{Z}$. The domain size is chosen to be $R_{\max }=50$ and $Z_{\max }=40$ to guarantee an unconfined radial boundary condition (see Appendix A) since such vortex flow is known to be very sensitive to confinement effects as experienced by Ruith et al. [7]. The solver is NEK5000 [30], which is based on a spectral element method defined on a Cartesian coordinate system $(X, Y, Z)$. Consequently, the DNSs are performed on a Cartesian grid and the results of this paper are presented in cylindrical coordinates $(R, \theta, Z)$, which is preferably used for swirling flows. Both coordinate systems are oriented in the trigonometric direction and share the same axial component $Z$ pointing inside the domain along the main axis of the cylinder. Their origins are located at the center of the inlet (see Fig. 1). The mesh contains 11040 hexahedral elements discretized using Gauss-Lobatto-Legendre and Gauss-Legendre points of polynomial order $P_{10}$ and $P_{8}$ to satisfy the inf-sup condition of the Navier-Stokes equations, and the time step defines a stable Courant-Friedrichs-Lewy (CFL) number of 0.37 (see Appendix A for a validation of this CFL choice). The initial conditions of the DNS are given by the Grabowski-Berger velocity profile (2) extended to the entire computational domain.

\section{B. Temporal-azimuthal Fourier decomposition}

The coherent flow patterns associated with the characteristic frequency of the DNS are extracted using a temporal-azimuthal Fourier decomposition. This decomposition is performed for the selected Reynolds values $\operatorname{Re}=[200,220,230,250]$. The coherent flow fluctuations $\mathbf{U}^{\prime}=\left(\mathbf{V}^{\prime}, P^{\prime}\right)$ are therefore obtained by subtracting the time-average flow $\overline{\mathbf{U}}$ of the instantaneous flow $\mathbf{U}$, yielding, after Fourier decomposition, the expression

$$
\begin{aligned}
\mathbf{U}^{\prime}\left(R, \theta, Z, t_{q}\right) & =\mathbf{U}\left(R, \theta, Z, t_{q}\right)-\overline{\mathbf{U}}(R, \theta, Z) \\
& =\operatorname{Re}\left(\sum_{m=0}^{\infty} \frac{1}{N} \sum_{k=0}^{N-1}\left[\mathbf{A}_{m q}(R, Z) e^{-i \omega_{k} t_{q}} \cos (m \theta)+\mathbf{B}_{m q}(R, Z) e^{-i \omega_{k} t_{q}} \sin (m \theta)\right]\right) \\
& =\operatorname{Re}\left(\sum_{m=0}^{M-1} \frac{1}{N} \sum_{k=0}^{N-1} \mathbf{U}_{m q}^{\prime}(R, Z) e^{i\left(m \theta-\omega_{k} t_{q}\right)}\right)
\end{aligned}
$$


where $\omega_{k}=k \Delta \omega=k 2 \pi / N$, with $N$ the number of samples. This definition leads to the relations $\operatorname{Re}\left(\mathbf{U}_{m q}^{\prime}\right)=\mathbf{A}_{m q r}=\mathbf{B}_{m q i}$ and $\operatorname{Im}\left(\mathbf{U}_{m q}^{\prime}\right)=\mathbf{A}_{m q i}=-\mathbf{B}_{m q r}$, with the index $r$ for real part and $i$ for imaginary part of $\mathbf{A}_{m q}$ and $\mathbf{B}_{m q}$. The azimuthal decomposition is computed on the fly during the DNS using the FFTW package [31] embedded in NEK5000 [30]. At each time step, part of the domain from $R=3$ to $Z=30$ is interpolated on a cylindrical distribution of points using the spectral interpolation of NEK5000 with $10^{-13}$ accuracy. An accurate Fourier decomposition of the flow involved 16 azimuthal points, leading to $M=16$ modes and a time series of $N=3.2 \times 10^{5}$ samples for cases with a low frequency, that represent a dimensionless time of $1.6 \times 10^{3}$. The frequency spectrum associated with the temporal decomposition is then computed with MATLAB fast Fourier transform. As the modes are extracted during the DNS, this analysis can be viewed as an a posteriori description of the flow modes.

\section{Global stability analysis}

The small disturbance dynamics can be viewed as an a priori description of the coherent flow patterns by investigating the exponential growth of infinitesimal perturbations from an initial state. Global linear stability analyses around the time-average flow field $\overline{\mathbf{U}}=(\overline{\mathbf{V}}, \bar{P})$ of the Navier-Stokes equations were performed. Such an analysis is found relevant to predict the frequency of limit cycle dynamics above the instability threshold in open flows presenting self-sustained oscillations. In contrast, global stability analysis around the base flow determines accurately the instability threshold but often fails to predict the correct frequencies (see, e.g., [32-35]).

In the present study, instead of computing an expansive 3D analysis, we take advantage of the geometry and flow symmetry to compute the linear stability analysis in a $2 \mathrm{D}$ axisymmetric domain $\Omega_{a}$, i.e., the meridional plane of the $3 \mathrm{D}$ domain $\Omega$. Infinitesimal perturbations are therefore decomposed in normal mode expansion with respect to the time $t$ and the azimuthal coordinate $\theta$ as

$$
\mathbf{U}^{\prime}(R, \theta, Z, t)=\mathbf{u}(R, Z) \exp [i(m \theta-\omega t)]+\text { c.c. },
$$

with $m \in \mathcal{Z}$ the azimuthal wave number, $\omega$ the eigenvalue, and c.c. the complex conjugate. Assuming that the mean flow is unperturbed by the Reynolds stresses at the first order (see $[32,36,37]$ ), the ensuing eigenvalue problem is written in a compact form as

$$
\left[-i\left(\omega_{r}+i \omega_{i}\right) \mathcal{N}+\mathcal{L}_{m}(\overline{\mathbf{U}})\right] \mathbf{u}=\mathbf{0} \text { in } \Omega,
$$

similar to global stability analysis around the base flow, where $\mathcal{L}_{m}$ is the operator for the linearized Navier-Stokes equations of azimuthal wave number $m$ and $\mathcal{N}$ is the singular operator premultiplying the time derivative.

The involvement of this assumption with the correct shape and the frequency of the linear global mode from the global stability analysis around the mean flow is further discussed in Refs. [33,34]. Shape and frequency deviations are reported as soon as the harmonics of the limit cycle contribute to the system dynamics or as other modes are harmonically associated with the dominant mode. Unfortunately, such properties cannot be determined a priori and nonlinear numerical simulations are required to determine the harmonic dependence of the limit cycle solution. The correction of the linear stability analysis by higher-order terms remains an open question (see Ref. [38] for a recent contribution in that direction), which is beyond the scope of the present study. However, excellent agreement with the frequency is reported for the present configuration using Eq. (6) (see Ref. [35]).

The associated boundary conditions of Eq. (6) are a zero disturbance at the inlet $\Gamma_{\text {in }}$ and a free outflow on the external boundary $\Gamma_{\text {ext }}$. The boundary conditions on $\Gamma_{\text {axis }}$ which depend on the azimuthal wave number [39] are summarized in Table I. The convective boundary on $\Gamma_{\text {out }}$ is converted to a free-outflow condition without impacting the solution of the eigenvalue problem (see Appendix A).

The global stability analysis is carried out by a finite-element method implemented in the FREEFEM ++ software [40]. The weak form of the eigenvalue problem is derived after being premultiplied by the radial coordinate $R$ to avoid singularities on the symmetry axis. The eigenvalue 
TABLE I. Boundary conditions on the axisymmetric axis applied to the disturbances for different azimuthal wave numbers.

\begin{tabular}{lc}
\hline \hline Azimuthal wave number & Boundary conditions \\
\hline$m=0$ & $v_{R}=v_{\theta}=0, \partial_{R} v_{Z}=0$ \\
$m= \pm 1$ & $v_{Z}=p=0, \partial_{R} v_{R}=\partial_{R} v_{\theta}=0$ \\
$m>1$ & $v_{R}=v_{\theta}=v_{Z}=0$ \\
\hline \hline
\end{tabular}

and eigenvectors are computed using the implicit restarted Arnoldi method of the ARPACK library [41] with a tolerance of $10^{-6}$. The 2D axisymmetric mesh is made by approximately 400000 Taylor-Hood triangular elements of $P_{2}-P_{1}$ shape functions. The meridional plane of the time-average flow from the 3D DNS, which is computed on the fly in NEK5000, is interpolated on the 2D axisymmetric mesh using the spectral interpolation tool of NEK5000, accurate at $10^{-13}$.

\section{INSTANTANEOUS FLOW}

Three-dimensional representations of the vortex flow, made visible by isocontours of axial vorticity of the DNSs, are displayed in Fig. 2. They illustrate the time history of the flow patterns observed at a Reynolds value of $\operatorname{Re}=220$ and for a swirl number of $S=1.095$. These snapshots show a recirculation bubble right after the inlet, the so-called bubble vortex breakdown with additional vortical flow structures in the wake of this bubble. In the first illustration [Fig. 2(a) at $t=2278$ ], the bubble is followed by a single-helix labeled primary spiral which is typical of the spiral vortex breakdown phenomenon. This spiral indeed spins temporally in the same direction as the inlet vortex rotation and coils spatially in the opposite direction, consistent with the previous results of Ruith et al. [7]. The time history of this primary spiral shows a modulation of vorticity intensity, which becomes stronger at the time step $t=2289$ [Fig. 2(b)] than at the later time step $t=2341$ [Fig. 2(g)].

Furthermore, a second vortical structure labeled downstream spiral is observed at this Reynolds value and starts to be materialized by vorticity isocontours at the time step $t=2330$ [Fig. 2(c)], around $Z=10$. This downstream vortex develops separately from the primary spiral between time steps $t=2330$ [Fig. 2(c)] and $t=2368$ [Fig. 2(g)]. It appears with thinner and elongated vorticity isocontours at the latter time step.

Finally, a third vortical structure labeled near-wake spiral is further observed in the near wake of the bubble, which is well defined at the time step $t=2341$ [Fig. 2(d)]. While this vortex temporally spins in this near-wake location, it has the particular feature of reattaching to the downstream spiral at time $t=2356$ [Fig. 2(e)] and detaching in the next snapshot [Fig. 2(f)]. This selected example shows the rich dynamics of this swirling wake flow. The characterization of its nonlinear dynamics represents the core of the present study.

\section{BIFURCATION ANALYSIS}

A global picture of the dynamics of this swirling wake flow is depicted in Fig. 3. This bifurcation diagram is shown for a fixed swirl number $S=1.095$ and up to a Reynolds value of $\operatorname{Re}=300$. Based on the time series data monitored at the location where the three vortical structures previously described in Sec. IV meet, $(R, \theta, Z)=(0.1,0.0,6.0)$, this bifurcation diagram is built by collecting and displaying each maximum and minimum of the radial velocity oscillations after the system reaches an established state. For this purpose, transient effects are excluded by a qualitative check of time series ensuring that full amplitude and coherence of the velocities are established. Using this technique, fixed points are represented by a single point, while higher-dimensional states have either a finite number or a finite approximation of an infinite number of points, as a consequence of the finite length of the time series. In particular, a stable limit cycle has two repeated points (one repeated peak and one repeated trough), while a quasiperiodic cycle on a $k$-torus $\mathbb{T}_{k}$ (with $k$ incommensurable 

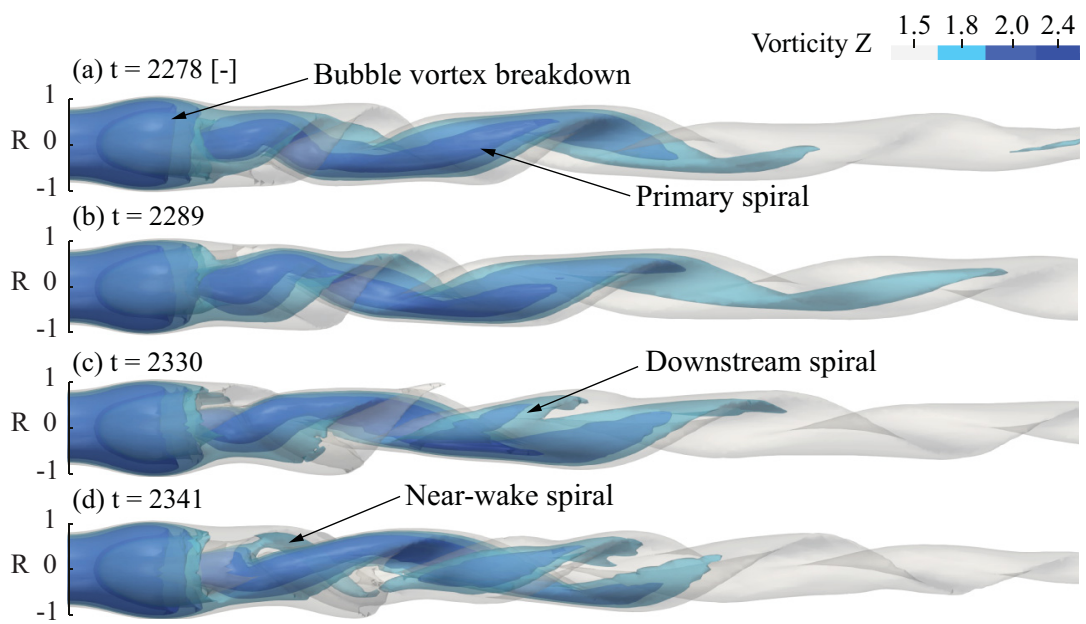

(e) $\mathrm{t}=2356$

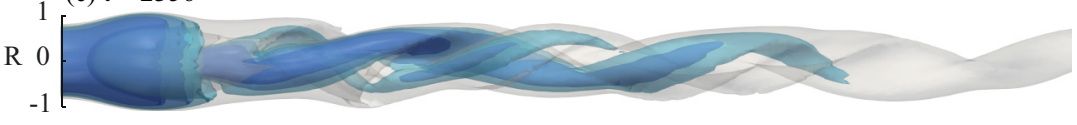

(f) $t=2362$
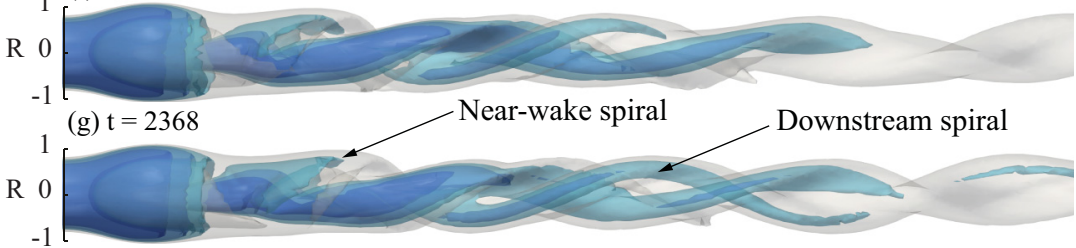

(h) $\mathrm{t}=2378$

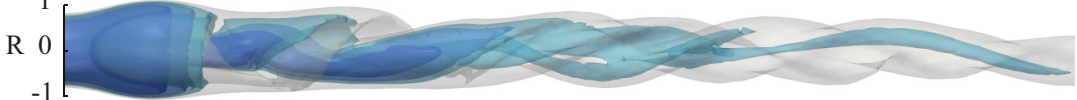

(i) $\mathrm{t}=2399$

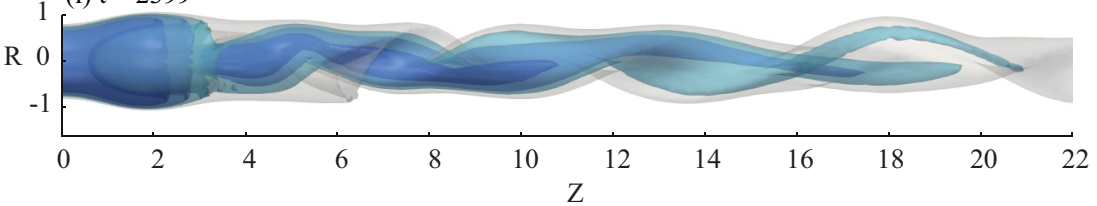

FIG. 2. Time history of the swirling flow induced by a Grabowski-Berger vortex for a swirl number of $S=1.095$ and a Reynolds number of $\operatorname{Re}=220$, made visible by axial vorticity isocontours.

frequencies) and a chaotic regime have spread out points bounded within a range of $V_{R}$ in the present case.

At low Reynolds number, the system is attracted toward a fixed-point solution, defined as the base flow of the governing equations (1), and it occurs for a Reynolds value up to $\operatorname{Re}=143.5$, as reported by Pasche et al. [35]. A supercritical Hopf bifurcation is then observed leading to a limit cycle solution below $\operatorname{Re} \simeq 200$. Above $\operatorname{Re}=200$, several minimum and maximum values are observed, implying that the system is attracted toward higher-dimensional states. The range of radial velocity $V_{R}$ increases up to $\mathrm{Re}=230$, where the maxima and minima are mixed, and leads to a dense line of points in the present diagram (Fig. 3). The system turns back into a limit cycle solution between $\operatorname{Re}=240$ and $\operatorname{Re}=255$, characterized by two repeated points. This limit cycle loses its stability above $\operatorname{Re}=255$. A second densification of the minimum and maximum values, which characterizes 


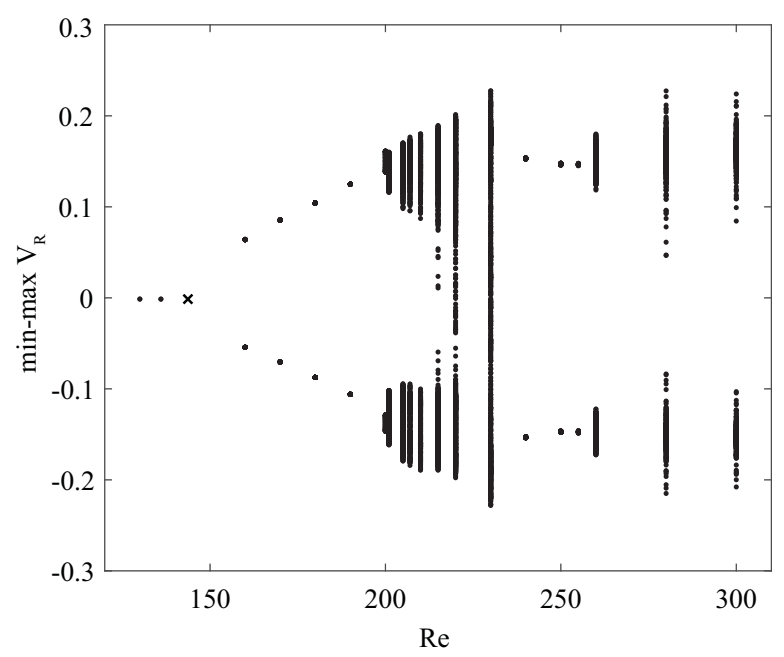

FIG. 3. Bifurcation diagram of the min-max temporal series of the radial velocity $V_{R}$ at $(R, \theta, Z)=$ $(0.1,0.0,6.0)$ (point markers). The instability threshold of Pasche et al. [35], at $\operatorname{Re}=143.5$, is illustrated as a cross.

quasiperiodic or chaotic oscillations, is then observed and seems to persist over larger Reynolds values, although it is not further studied here.

\section{NONLINEAR TIME SERIES ANALYSIS}

\section{A. Nonlinear time series}

Nonlinear time series of the established tangential velocity monitored at the location $(R, \theta, Z)=(0.1,0.0,6.0)$ are displayed in Fig. 4 for the selected Reynolds values $\operatorname{Re}=$ $[180,200,205,215,220,230,250,300]$. The stable limit cycle arising from a supercritical Hopf bifurcation associated with the spiral vortex breakdown is observed for $\mathrm{Re}=180$ in Fig. 4(a). This periodic oscillation appears with the development of an unstable mode $[7,8]$, which saturates by the nonlinearity of the governing equations and leads to a finite-amplitude oscillation of the flow. The system is then attracted toward a regime with two frequencies at $\mathrm{Re}=200$ observed by the signal modulation of the time series [see Fig. 4(b)]. Between the Reynolds values of $\operatorname{Re}=205$ and $R e=230$ [Figs. 4(c)-4(f)], an additional low oscillation slots in the system dynamics, which presents irregular oscillations between $\operatorname{Re}=215$ and $\operatorname{Re}=220$ [Figs. 4(d) and 4(e)]. These higher system states are followed by a synchronization regime, as illustrated at a Reynolds value of $\mathrm{Re}=250$ in Fig. $4(\mathrm{~g})$. After this synchronization regime, the flow becomes quasiperiodic or chaotic from $\mathrm{Re}=260$ until $\operatorname{Re}=300$ with the presence of long-time dynamics.

\section{B. Sensitivity to initial disturbance}

Sensitivity to initial disturbances is investigated to clarify the dynamical state at Reynolds values of $\operatorname{Re}=[205,230]$ and $\operatorname{Re}=[215,220]$ where the dynamics are patterned by low frequencies, with irregular oscillations for the latter. The separation distance $\|D\|$, assessed by the instantaneous $L_{2}$-norm of the velocity at the monitoring point $(R, \theta, Z)=(0.1,0.0,6.0)$, is computed on two flow solutions: a reference solution and a disturbed solution which is initiated by a random bulk disturbance of amplitude $10^{-8}$ added to the reference solution. The disturbance is initialized at a selected time of the established state of the system. We should mention that the DNS flow distribution from a restarted 

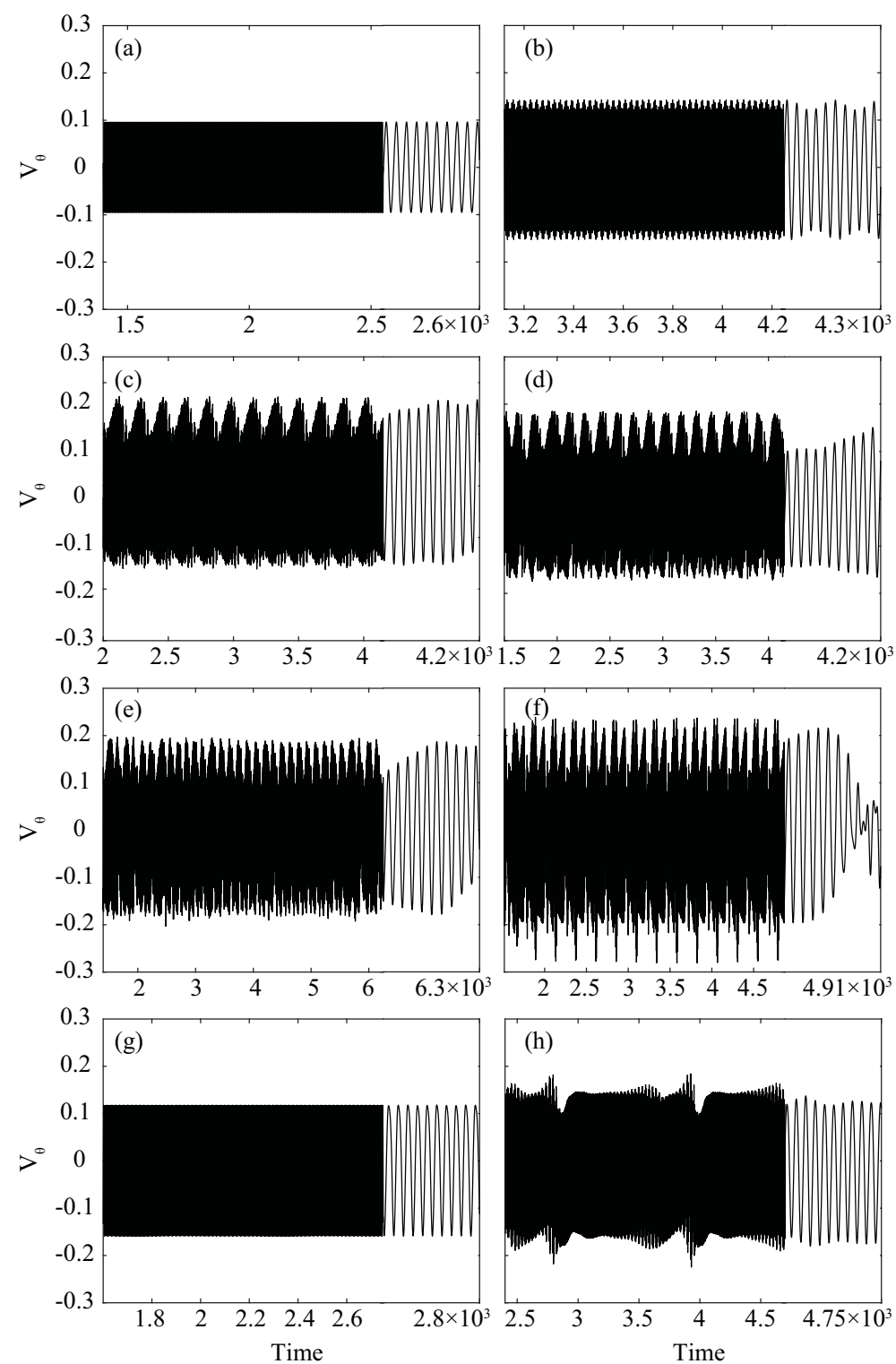

FIG. 4. Time series of the tangential velocity monitored at $(R, \theta, Z)=(0.1,0.0,6.0)$ for a swirl number of $S=1.095$ and selected Reynolds numbers (a) $\operatorname{Re}=180$, (b) $\operatorname{Re}=200$, (c) $\operatorname{Re}=205$, (d) $\operatorname{Re}=215$, (e) $\operatorname{Re}=220$, (f) $\operatorname{Re}=230$, (g) $\operatorname{Re}=250$, and (h) $\operatorname{Re}=300$. The end of each time series uses a zoomed scale.

solution remains invariant and the high accuracy of the spectral element method of NEK5000 allows us to carry out such an approach.

The time series of the reference and disturbed flows are presented in Fig. 5 for the Reynolds values of $\operatorname{Re}=[215,220]$. The separation of the two systems is visible after a time of $t=8.2 \times 10^{3}$ for the Reynolds value of $\operatorname{Re}=215$ and a time of $t=7 \times 10^{3}$ for the Reynolds value of $\operatorname{Re}=220$. 

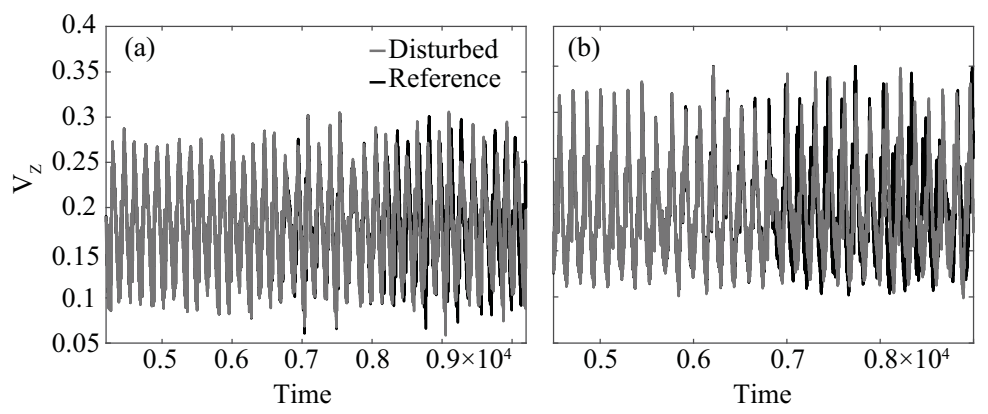

FIG. 5. Time series of the reference flow and the disturbed flow at Reynolds values of (a) $\operatorname{Re}=215$ and (b) $\operatorname{Re}=220$ for the monitoring points $(R, \theta, Z)=(0.1,0.0,6.0)$. The disturbed flow starts with random noise of amplitude $10^{-8}$ at the time step displayed in the graph.

The separation distance $\|D\|$ of these signals is displayed in Fig. 6 for the previous Reynolds values. At Reynolds values of $\mathrm{Re}=[205,230]$ [Figs. 6(a) and 6(d)], the separation distance remains around $10^{-5}$, meaning that the system is quasiperiodic even if an additional low frequency characterizes the time series [see Figs. 4(c) and 4(f)]. In contrast, an exponential separation of the flow solutions is reported at Reynolds values of $\operatorname{Re}=215$ and $\mathrm{Re}=220$ [Figs. 6(b) and 6(c)]. In the former case, a constant exponential coefficient can be extracted, while in the latter case it is only approximate due to a step change in the curve. Their exponential growths are close to zero, $\alpha=6.0 \times 10^{-5}$, and
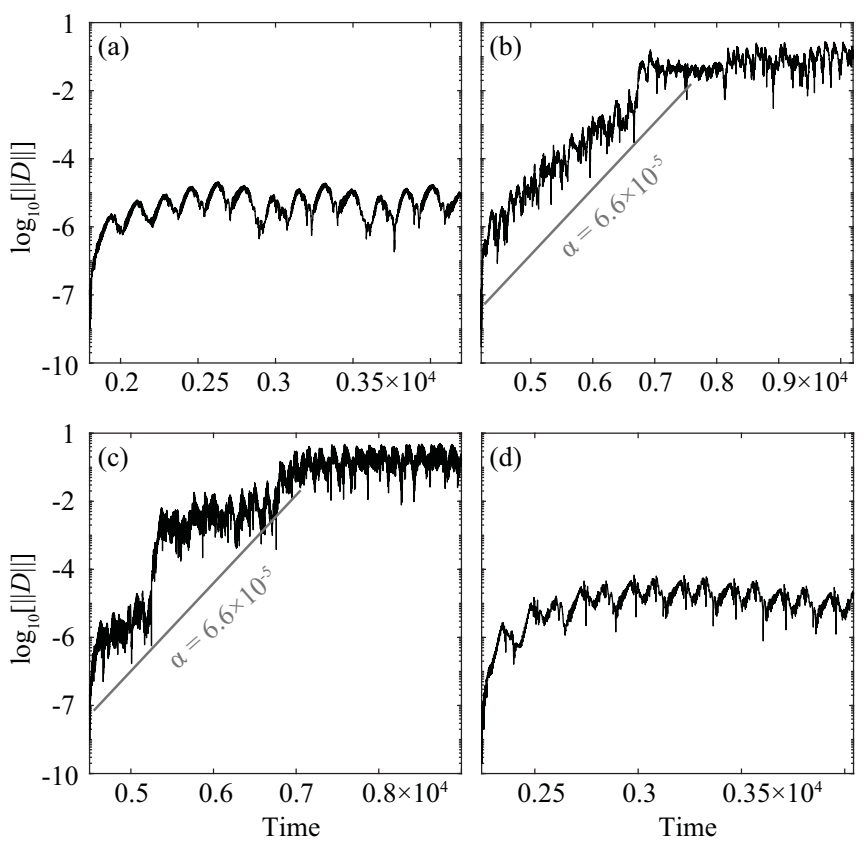

FIG. 6. The $L_{2}$-norm of the distance between the reference and disturbed dynamical state at the location $(R, \theta, Z)=(0.1,0.0,6.0)$ at Reynolds values of (a) $\operatorname{Re}=205$, (b) $\operatorname{Re}=215$, (c) $\operatorname{Re}=220$, and (d) $\operatorname{Re}=230$. 

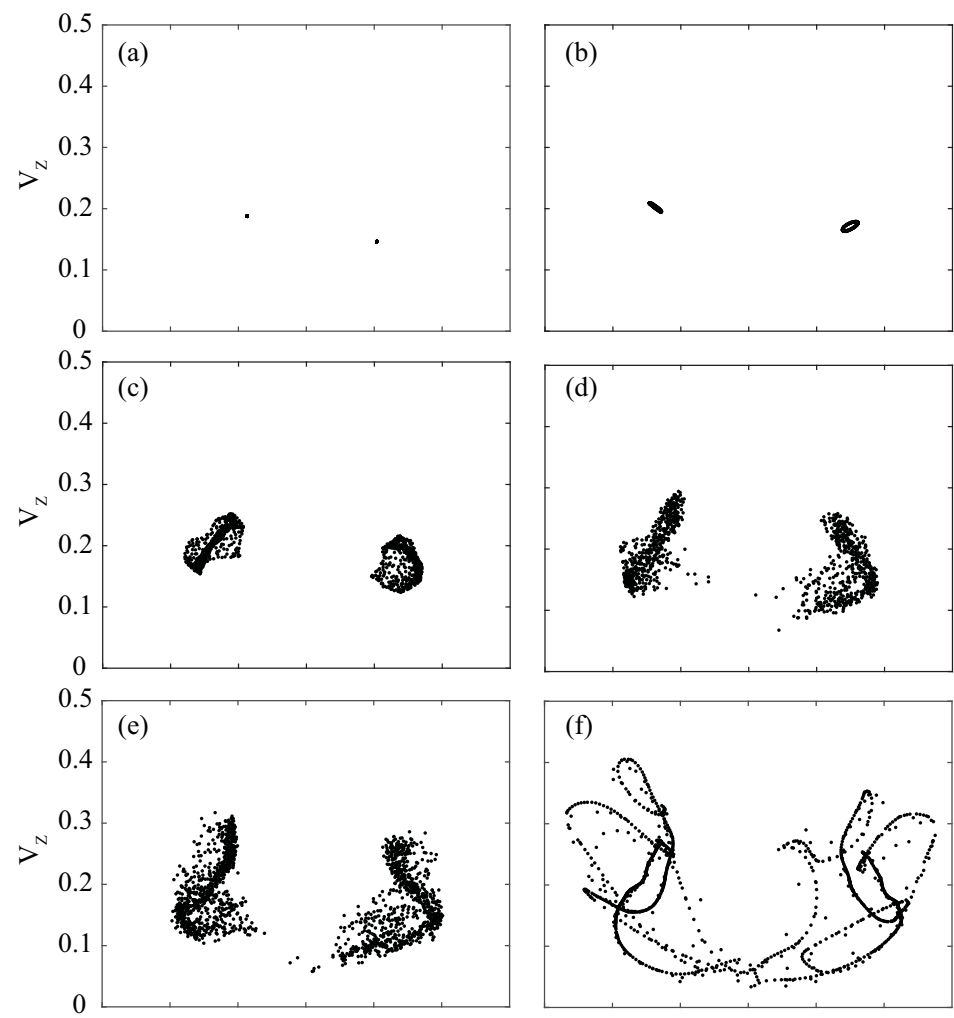

(f)
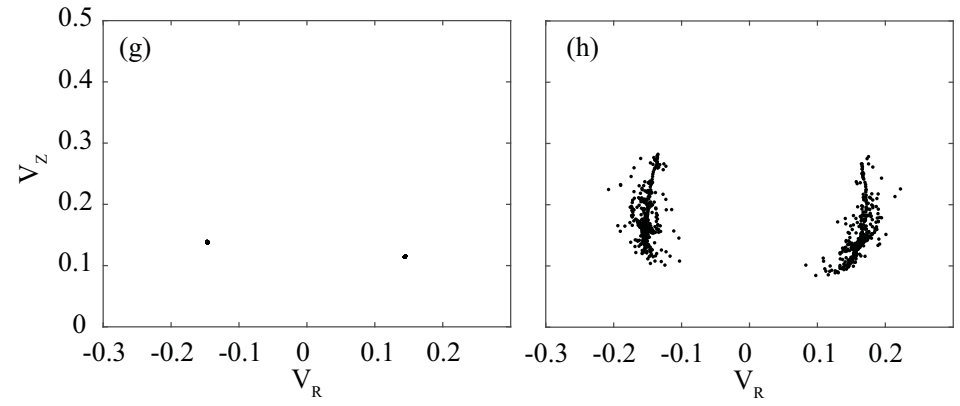

FIG. 7. Attractor cross section of the velocity time series at $(R, \theta, Z)=(0.1,0.0,6.0)$ for $V_{\theta}=0.001$, a swirl number of $S=1.095$, and selected Reynolds numbers (a) $\operatorname{Re}=180$, (b) $\operatorname{Re}=200$, (c) $\operatorname{Re}=205$, (d) $\operatorname{Re}=215$, (e) $\operatorname{Re}=220$, (f) $\operatorname{Re}=230$, (g) $\operatorname{Re}=250$, and (h) $\operatorname{Re}=300$.

nevertheless positive, which supports the finding that these systems tend to chaotic attractors at these Reynolds numbers $\operatorname{Re}=[215,220]$.

\section{Attractor cross sections}

Attractor cross sections of the time series at the monitoring point $(R, \theta, Z)=(0.1,0.0,6.0)$ are displayed in Fig. 7 for the selected Reynolds values $\mathrm{Re}=[180,200,205,215,220,230,250,300]$. These attractor cross sections were obtained by sampling the radial and axial velocity components associated with the selected tangential velocity component of value $V_{\theta}=0.001$. We therefore display cross sections of the phase space, which is useful to define the topological structure of 
attractors. Attractor cross sections are similar to Poincaré section maps except that a double number of points are plotted because both crossing directions are extracted, while only one direction is reported for a Poincaré section map. Limit cycles are represented as two points in the attractor cross section that is typically observed for Reynolds values of $\operatorname{Re}=180$ and $\operatorname{Re}=250$, in Figs. 7(a) and 7(g), respectively. The former Reynolds value is associated with the dynamics of the spiral vortex breakdown and the latter Reynolds value corresponds to the frequency synchronization state of the system.

A quasiperiodic regime with two incommensurable frequencies is represented by a closed path of the attractor cross section, typically at a Reynolds value of $\mathrm{Re}=200$ and in a more distorted way at $\operatorname{Re}=230$. The chaotic regimes identified at $\operatorname{Re}=[215,220]$ have dense cross sections [Figs. 7(d) and $7(\mathrm{e})$ ] because the correlation dimension of chaotic attractors is no longer an integer, like fractals (see Ref. [42]). The same structure of the attractor cross section is observed at $\operatorname{Re}=300$ [Fig. 7(h)], which is probably chaotic. At $\operatorname{Re}=205$ [Fig. 7(c)], a dense but bounded attractor cross section is reported. Since initial disturbances relax back towards the system's established state [see Fig. 6(a)], we suspect a stable quasiperiodic regime on a torus $\mathbb{T}_{3}$ for $\operatorname{Re}=205$.

\section{Amplitude Fourier spectrum}

Amplitude Fourier spectra of the time series at the location $(R, \theta, Z)=(0.1,0.0,6.0)$ and snapshots of DNS axial vorticity isocontours for the selected bifurcation parameters are presented in Fig. 8. In addition, movies of this flow for each value of the bifurcation parameter investigated are available in the Supplemental Material [43].

At $\operatorname{Re}=180$, the periodic signal of fundamental frequency $f_{1}$ is not purely sinusoidal; its first harmonic appears in the frequency spectrum, labeled $f_{a}$ in Fig. 8(a). The associated vorticity isocontours displayed in Fig. 8(b) show the axisymmetric recirculation bubble and a well-defined single spiral spinning in the bubble wake, typical of the spiral vortex breakdown and called primary spiral in the present study.

At $\operatorname{Re}=200$, the snapshot in Fig. 8(d) depicts a tilted recirculation bubble resulting from the oscillations of the bubble around the symmetry axis of the domain. In the bubble wake, the primary spiral shows a higher vorticity intensity than at $R e=180$. In addition, a second vortex appears at a downstream location $Z=12$, called a downstream spiral. This vortex is made visible by a vorticity isocontour of value 1.8 and has a weaker vorticity magnitude than the primary spiral. This downstream spiral is associated with the frequency $f_{2}$ in the Fourier amplitude spectrum [Fig. 8(c)]. The birth of this second frequency turns the system into a quasiperiodic state on a torus $\mathbb{T}_{2}$, with two incommensurable frequencies $f_{1} / f_{2}=1.2667$. The quadratic nonlinear interaction of the primary and downstream spirals generates a frequency of smaller amplitude labeled $f_{b}=f_{1}-f_{2}$ in Fig. 8(c). The frequency shift between these two spirals means that they periodically merge and separate, due to the predominant intensity of the primary spiral as seen in the movie of [43].

A torus $\mathbb{T}_{3}$ appears at $\operatorname{Re}=205$ by the development of a third frequency peak in the Fourier amplitude spectrum, labeled $f_{3}$ in Fig. 8(e). Three incommensurable frequencies $f_{1} / f_{3}=1.0294$, $f_{1} / f_{2}=1.2666$, and $f_{2} / f_{3}=0.8127$ are therefore identified in this quasiperiodic attractor, which appears to be stable in view of its sensitivity to initial disturbance [Fig. 6(a)]. Nonlinear interactions between these modes produce different frequencies (among all we have labeled $f_{c}=f_{1}-f_{3}$ and $f_{d}=2 f_{1}-f_{3}$ ), which consequently induce the slow modulation observed in the time series [Fig. 4(c)]. The snapshot of vorticity isocontours shows again the primary spiral and the downstream spiral associated with the frequency peaks $f_{1}$ and $f_{2}$. However, no clearly visible vortical structure is identified for the frequency peak $f_{3}$ [see Fig. 8(f)].

The chaotic attractors at $\mathrm{Re}=[215,220]$ [Figs. $8(\mathrm{~g})$ and $8(\mathrm{i})]$ result from the destabilization of the torus $\mathbb{T}_{3}$ woven by these three incommensurable frequencies. Quasiperiodicity turns into a chaotic regime as the second frequency peak $f_{2}$ becomes closer to the first $f_{1}$ and third $f_{3}$ frequency peaks. At $\operatorname{Re}=220$ [Fig. 8(j)], several helical vortices are observed, the primary spiral of strong amplitude, the downstream spiral that now starts from $Z=6.5$, and a near-wake spiral located behind the bubble 

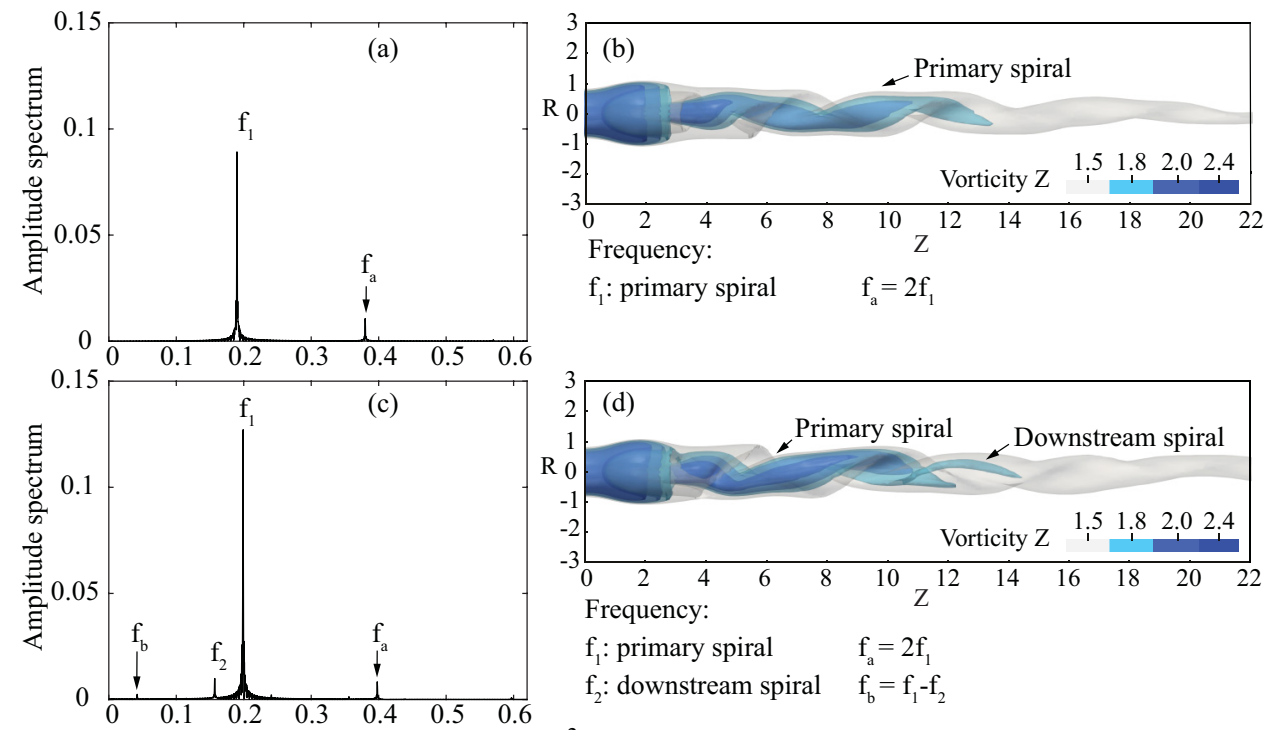

$\begin{array}{ll}\mathrm{f}_{1} \text { : primary spiral } & \mathrm{f}_{\mathrm{a}}=2 \mathrm{f}_{1} \\ \mathrm{f}_{2} \text { : downstream spiral } & \mathrm{f}_{\mathrm{b}}=\mathrm{f}_{1}-\mathrm{f}_{2}\end{array}$
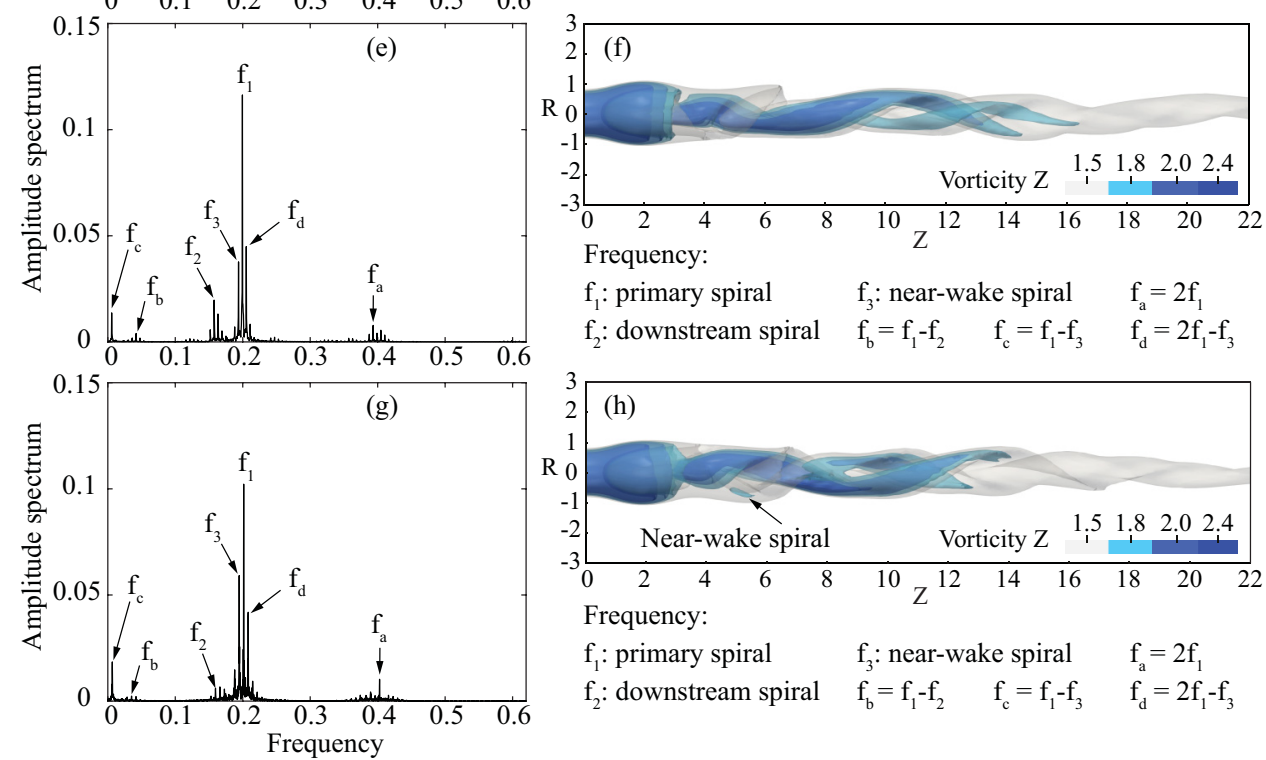

FIG. 8. Fast Fourier transform of the tangential velocity time series $V_{\theta}$ at $(R, \theta, Z)=(0.1,0.0,6.0)$ for a swirl number of $S=1.095$ and Reynolds numbers (a) and (b) $\operatorname{Re}=180$, (c) and (d) $\operatorname{Re}=200$, (e) and (f) $\operatorname{Re}=205$, (g) and (h) $\operatorname{Re}=215$, (i) and (j) $\operatorname{Re}=220$, (k) and (l) $\operatorname{Re}=230,(\mathrm{~m})$ and (n) $\operatorname{Re}=250$, and (o) and (p) $\operatorname{Re}=300$ and snapshots of the associated vorticity isocontours.

at $Z=5$, which are phase shifted compared to the primary spiral. These three vortical structures evolve in time according to the following sequence: While the bubble and the primary spiral keep spinning at a fixed location, the downstream and near-wake spirals start growing and attach together, as displayed in Fig. 2(i), before being embedded in the primary vortex cyclically. A movie of this sequence is available in [43].

Three successive Hopf bifurcations attracting the system towards a quasiperiodic regime on a torus $\mathbb{T}_{3}$ with three incommensurable frequencies can become unstable and lead to a chaotic regime. This scenario is a typical Ruelle-Takens-Newhouse route to chaos, which has already appeared in several 

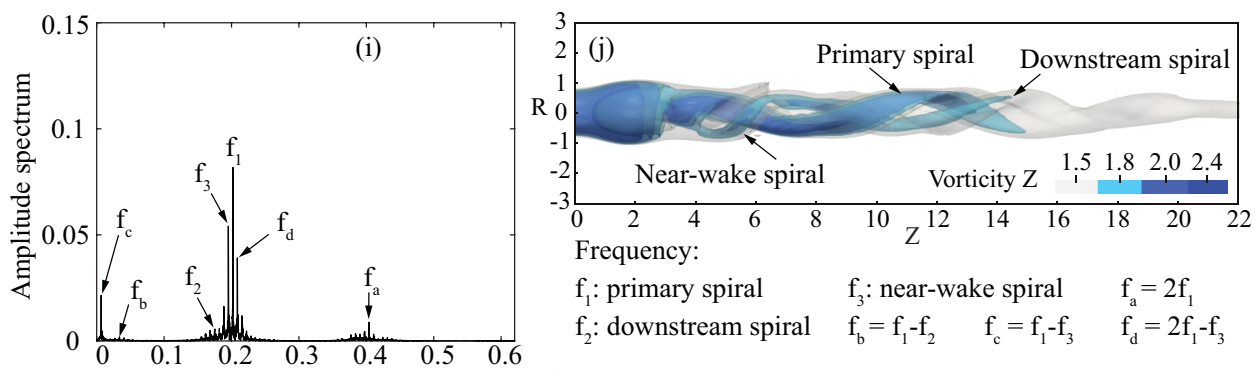

Frequency:

$\mathrm{f}_{1}$ : primary spiral $\quad \mathrm{f}_{3}$ : near-wake spiral $\quad \mathrm{f}_{\mathrm{a}}=2 \mathrm{f}_{1}$ $\mathrm{f}_{2}$ : downstream spiral $\mathrm{f}_{\mathrm{b}}=\mathrm{f}_{1}-\mathrm{f}_{2} \quad \mathrm{f}_{\mathrm{c}}=\mathrm{f}_{1}-\mathrm{f}_{3} \quad \mathrm{f}_{\mathrm{d}}=2 \mathrm{f}_{1}-\mathrm{f}_{3}$
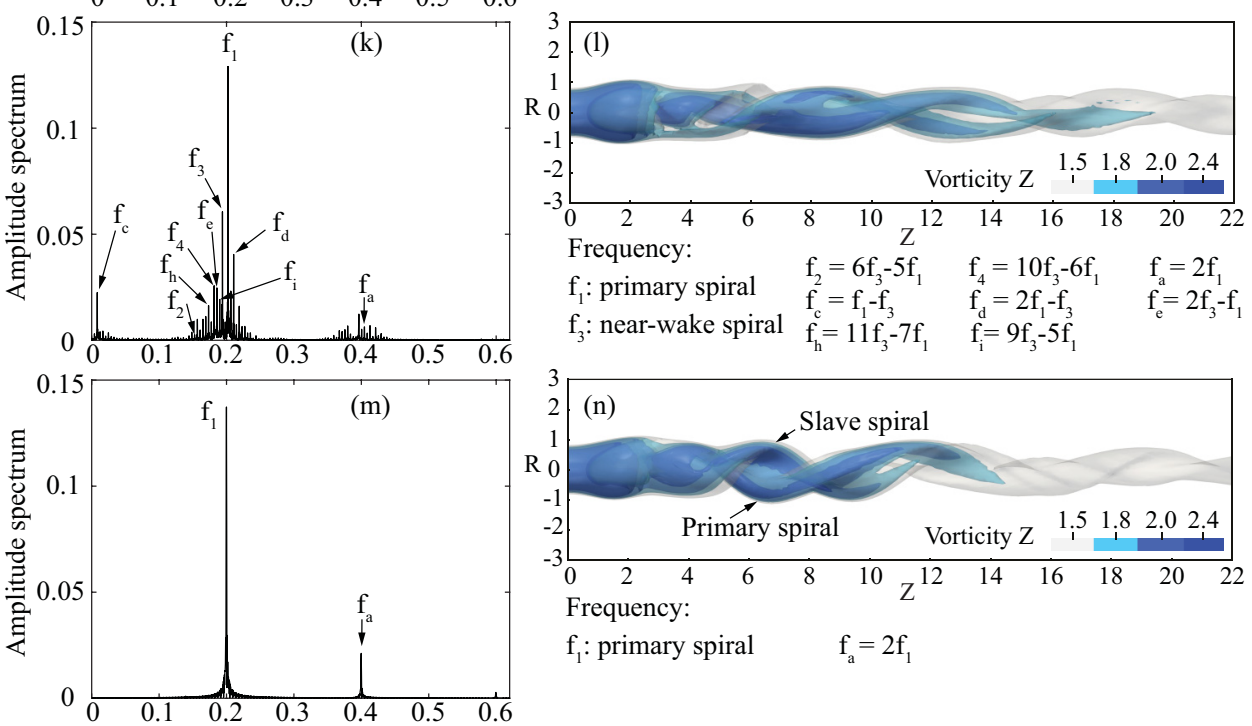

$\mathrm{f}_{1}$ : primary spiral $\quad \mathrm{f}_{\mathrm{a}}=2 \mathrm{f}_{1}$
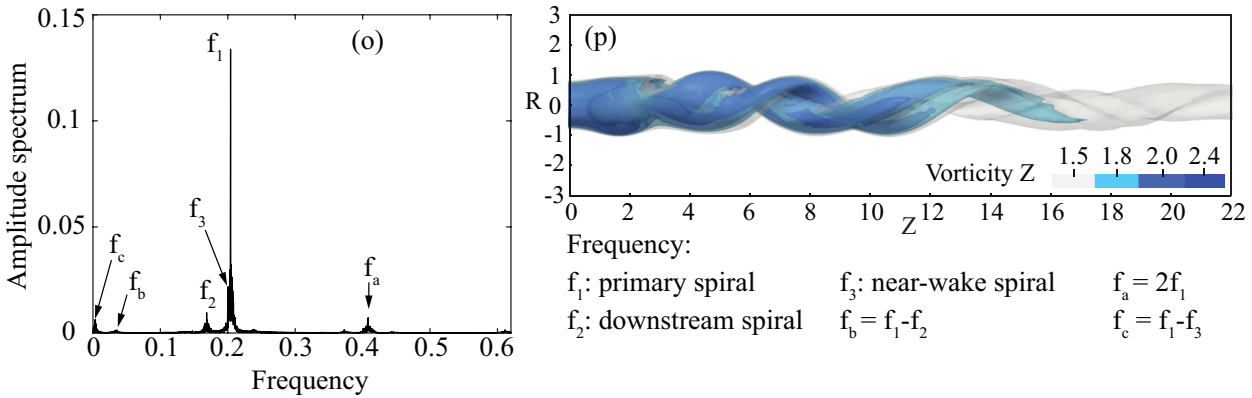

FIG. 8. (Continued.)

hydrodynamic examples (see, e.g., [18,20,25,44,45]). In particular, Oteski et al. [46] reported this route to chaos including a stable three-frequency torus $\mathbb{T}_{3}$ in a confined two-dimensional differential convection case. In the Ruelle-Takens-Newhouse scenario, the onset of the transition from a torus $\mathbb{T}_{3}$ to a strange attractor is not predictable [47]. Only the computation of the first three maximum Lyapunov exponents can strictly distinguish a stable torus $\mathbb{T}_{3}$ from an unstable one. The three largest Lyapunov exponents are equal to zero for a stable quasiperiodic $\mathbb{T}_{3}$ torus, which is in autonomous dissipative dynamical system [48], while at least one Lyapunov exponent must be positive for a chaotic state. In the present study, the Lyapunov exponents have not been computed due to the required computational time. However, sensitivity to the initial condition has been analyzed (Fig. 6) and based on these results, we expect a stable torus $\mathbb{T}_{3}$ observed at $\mathrm{Re}=205$. In addition, the transition from this torus $\mathbb{T}_{3}$ to the chaotic regime at $\mathrm{Re}=220$ is associated with the frequency 
migration of the downstream spiral $f_{2}$ to the primary spiral frequency $f_{1}$ and the near-wake spiral $f_{3}$, which emphasizes the nonlinear interactions.

The chaotic attractor turns into a torus $\mathbb{T}_{2}$ with two incommensurable frequencies for $\mathrm{Re}=230$ [Fig. 8(k)], as seen in the attractor cross section [Fig. 7(f)]. This state is indeed made possible by the fact that the second frequency becomes a subharmonic of $f_{1}$ and $f_{3}$ by the relation $f_{2}=6 f_{1}-5 f_{3}$. However, to obtain the peak density observed in the Fourier amplitude spectrum, a fourth frequency appears as commensurable with the first and third frequencies $f_{4}=10 f_{3}-6 f_{1}$. The quasiperiodic regime is therefore due to the two incommensurable frequencies $f_{1}$ and $f_{3}$, which govern the flow dynamics and drive the slaved modes of frequency $f_{2}$ and $f_{4}$. In Fig. $8(\mathrm{k})$, all the peaks of the Fourier amplitude spectrum are successfully identified by the use of these two frequencies (see Appendix B). A snapshot of axial vorticity contours corresponding to this amplitude Fourier spectrum is displayed in Fig. 8(1). While several frequencies appear in this spectrum, we can only identify the vortical structures based on the result of the previous Reynolds number $(\operatorname{Re}=220)$. The primary spiral has again the strongest vorticity and the near-wake spiral, in addition, attaches to the bubble crown and to the downstream spiral continuously at this time step.

The amplitude Fourier spectrum of the synchronization state observed at a Reynolds value of $\operatorname{Re}=250$ shows a well-defined peak $f_{1}$ and its associated harmonic $f_{a}=2 f_{1}$ [Fig. 8(m)]. The four frequencies observed in the quasiperiodic regime at $\mathrm{Re}=230$ are substituted for $f_{1}$ at $\operatorname{Re}=250$. The vorticity isocontours, displayed in Fig. 8(n), show not only a single spiral but two helical vortices: a primary spiral with a dense isocontour of value 2.4 , starting in the bubble wake, and a slaved spiral that has a modulated axial vorticity isocontour of value 2.4. Figure 8(n) shows that these two vortices are not in opposite phase but only slightly shifted. This indicates that this regime is not characterized by an $m=2$ double helix, as one could have deduced at first sight from streak lines or isosurfaces of vorticity, but rather by the coexistence of two $m=1$ modes as demonstrated in Sec. VII.

At $\operatorname{Re}=300$, the system turns into a chaotic regime identified by the dense attractor cross section [Fig. 7(h)], by the broad base of amplitude Fourier spectrum peaks, and by the identification of three incommensurable frequencies $f_{1}, f_{2}$, and $f_{3}$ [Fig. 8(o)]. The flow patterns displayed in Fig. 8(p) show two vortices similar to those observed at the previous Reynolds value $(\operatorname{Re}=250)$, which are not in opposite phase but rather phase shifted.

\section{MODE INTERACTIONS}

The nonlinear time series analysis provides the attractor specifications of this autonomous dissipative dynamical system at a local monitoring point for a range of Reynolds numbers. This local dynamics following from a stable attractor can be changed not only by the bifurcation parameter but also by vortical flow structures developing in a different spatial location of the domain as observed for the near-wake and downstream spirals. Since the present configuration consists of a semi-infinite unconfined domain and only pure hydrodynamic instabilities can generate nonlinear dynamics, the onset of these vortical structures is therefore further questioned by investigating the instability properties of the flow.

In our system, where supercritical Hopf bifurcations are the starting point of new branches, local linear temporal stability analysis characterizes the stability properties of these branches but incorrectly predicts the frequency and the vortical structure associated with this new stable branch [49]. Since the dominant advection direction of the flow causes the failure of this prediction, it should be addressed by evaluating the growth in not only time but also in the space of incoming fluctuations. This turns into the concept of the spatiotemporal stability theory of Huerre and Monkewitz [5], which distinguishes the situation where the advection dominates over growth and where instability waves are swept away while they grow (convective instability) from the one where growth dominates over advection and some instability waves withstand the advection and invade the entire domain (absolute instability). Both are able to modify attractors along the advection direction. Typically, Gallaire et al. [8] and Qadri et al. [11] identified two absolute regions in spiral vortex 


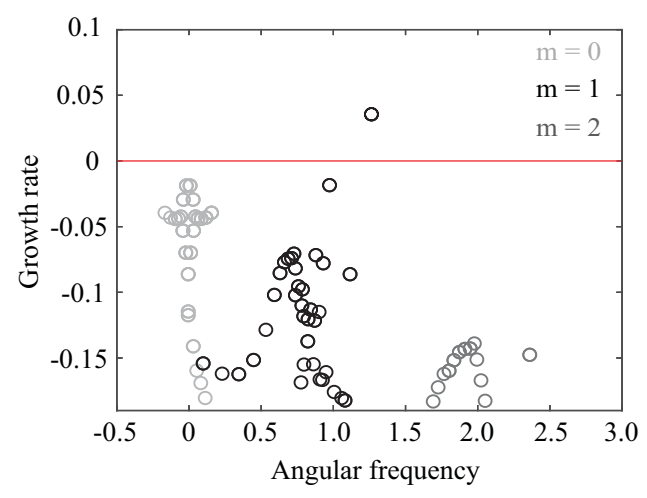

FIG. 9. Eigenvalue spectra obtained from the global stability analysis around the mean flow at $\mathrm{Re}=230$ for azimuthal wave numbers $m=0,1,2$.

breakdown with two distinct frequencies: one frequency on the recirculation bubble and the second frequency on the bubble wake.

However, for strongly nonparallel flows, a so-called global stability analysis is preferably used instead of a local stability analysis, which computes the instability properties of a collection of velocity profiles at successive local streamwise stations. Globally unstable flows behave therefore as flow oscillators, while globally stable flows are best studied through the response to harmonic forcing or to initial conditions (both referred to as nonmodal analyses). Several globally unstable eigenmodes will act as coupled flow oscillators with dedicated frequencies and their spatial core, sometimes called a wave maker [50], can induce different flow regimes along the streamwise direction, similar to a dynamical system subject to time delay [51]. Identifying the frequencies observed in the local amplitude Fourier spectrum as the consequence of global flow patterns is therefore essential to understand the system dynamical regimes.

Consequently, a global stability analysis is performed to identify linear unstable disturbances developing from an established state. Such an analysis is generally focused on limit cycle dynamics for mean flows and is rarely applied to a dynamical system state with multiple frequencies, as is the case here. This analysis is complemented by a temporal-azimuthal Fourier series extraction of the nonlinear simulation results, following the same decomposition as in the global stability analysis [see Eqs. (4) and (5)].

\section{A. Stability analysis}

Global stability analysis around the time-averaged flow field is investigated by solving the eigenvalue problem of Eq. (6). Examples of the eigenvalue spectrum are displayed in Fig. 9 for a Reynolds value of $\operatorname{Re}=230$ and for the first three azimuthal wave numbers $m=0,1,2$. An unstable eigenvalue is found for the single-helical mode $m=1$, while the eigenvalues of the other azimuthal wave numbers are stable.

We have systematically compared the frequency of the eigenvalues, obtained by global stability analysis around the mean flow for azimuthal wave number $m=0,1,2$ against the frequency of the DNS obtained by a discrete Fourier transform of the time series. The results of this comparison are presented in Fig. 10. Only eigenmodes of azimuthal wave number $m=1$ are represented for the following two reasons. First, the eigenmodes of azimuthal wave number $m=2$ have no correlation with the first harmonic frequency of the amplitude Fourier spectrum (labeled $f_{a}$ in Fig. 8). Second, while two eigenmodes of the azimuthal wave number $m=0$ could have the correct structure, their frequencies differ from those of the DNS (labeled $f_{c}$ in Fig. 8). The global stability analysis of azimuthal wave number $m=1$ predicts successfully not only the primary spiral frequency $f_{1}$ but also the frequency of the downstream spiral $f_{2}$ [Fig. 10(a)], as labeled in the amplitude Fourier 

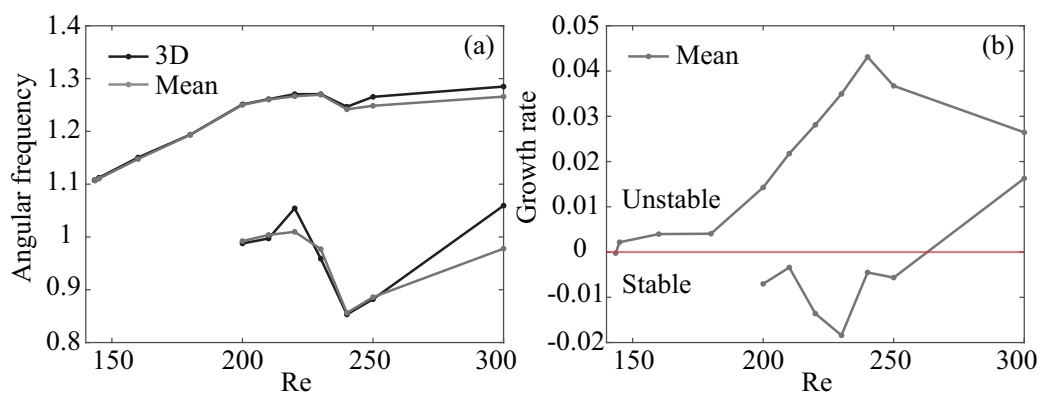

FIG. 10. Growth rate and angular frequency of the global linear stability of the mean flow for azimuthal wave number $m=1$ (labeled Mean) compared to the 3D angular frequency from the DNS (labeled 3D), computed by discrete Fourier transform at location $(R, \theta, Z)=(0.1,0.0,5.0)$.

spectrum of the time series (Fig. 8). The growth rates of these eigenmodes displayed in Fig. 10(b) show an unstable mode associated with the primary spiral $f_{1}$ as reported by Gallaire et al. [8], Meliga et al. [14], and Qadri et al. [11]. In contrast, the second eigenmode associated with the downstream spiral is stable up to a Reynolds value of $\operatorname{Re} \simeq 265$.

Axial velocity isocontours of these two $m=1$ eigenmodes are presented in Fig. 11 for a Reynolds number of $\operatorname{Re}=200$, normalized with respect to the $L_{2}$-norm of the semi-inner product. The primary eigenmode [Fig. 11(a)] shows two dissociated helices: the first helix at the beginning of the computational domain, which ends at $Z=12$, and the second helix downstream centered at $Z=14$. These helices contribute to the flow dynamics at the same frequency $f_{1}$. The downstream eigenmode [Fig. 11(b)] is centered at $Z=15$ and forms a single helix precessing at the frequency $f_{2}$ located downstream of the primary eigenmode.

Although the downstream spiral is found to be stable in the stability analysis, it finally develops and takes part in the dynamics of the system generating the frequency $f_{2}$. This second eigenmode has the same azimuthal wave number as the first one and we can therefore expect an indirect excitation mechanism to underpin this development.

Since only single-helical eigenmodes are found relevant, the system is governed by nonlinear interactions of single-helical modes. The synchronization state observed at $\mathrm{Re}=250$ is therefore associated with the concordance of two single spirals, while two vortical structures are observed, the primary spiral and the slave spiral [Fig. 8(n)].
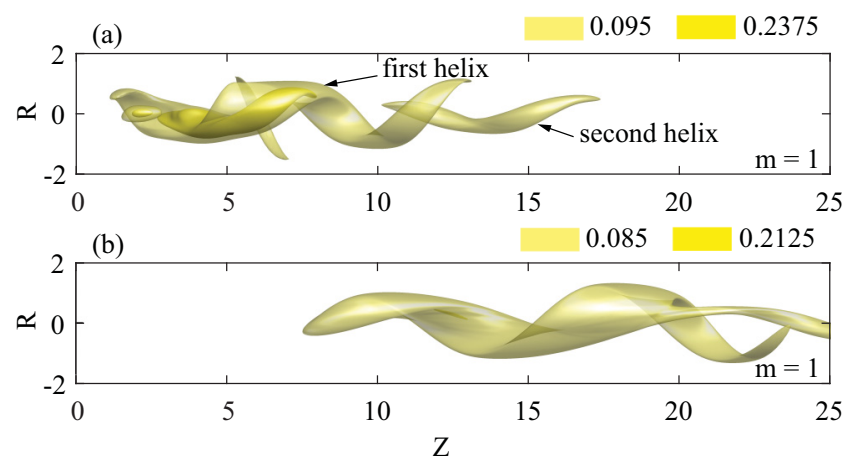

FIG. 11. Primary (a) and downstream (b) eigenmode axial velocity isocontours for a Reynolds value of $\operatorname{Re}=200$. 

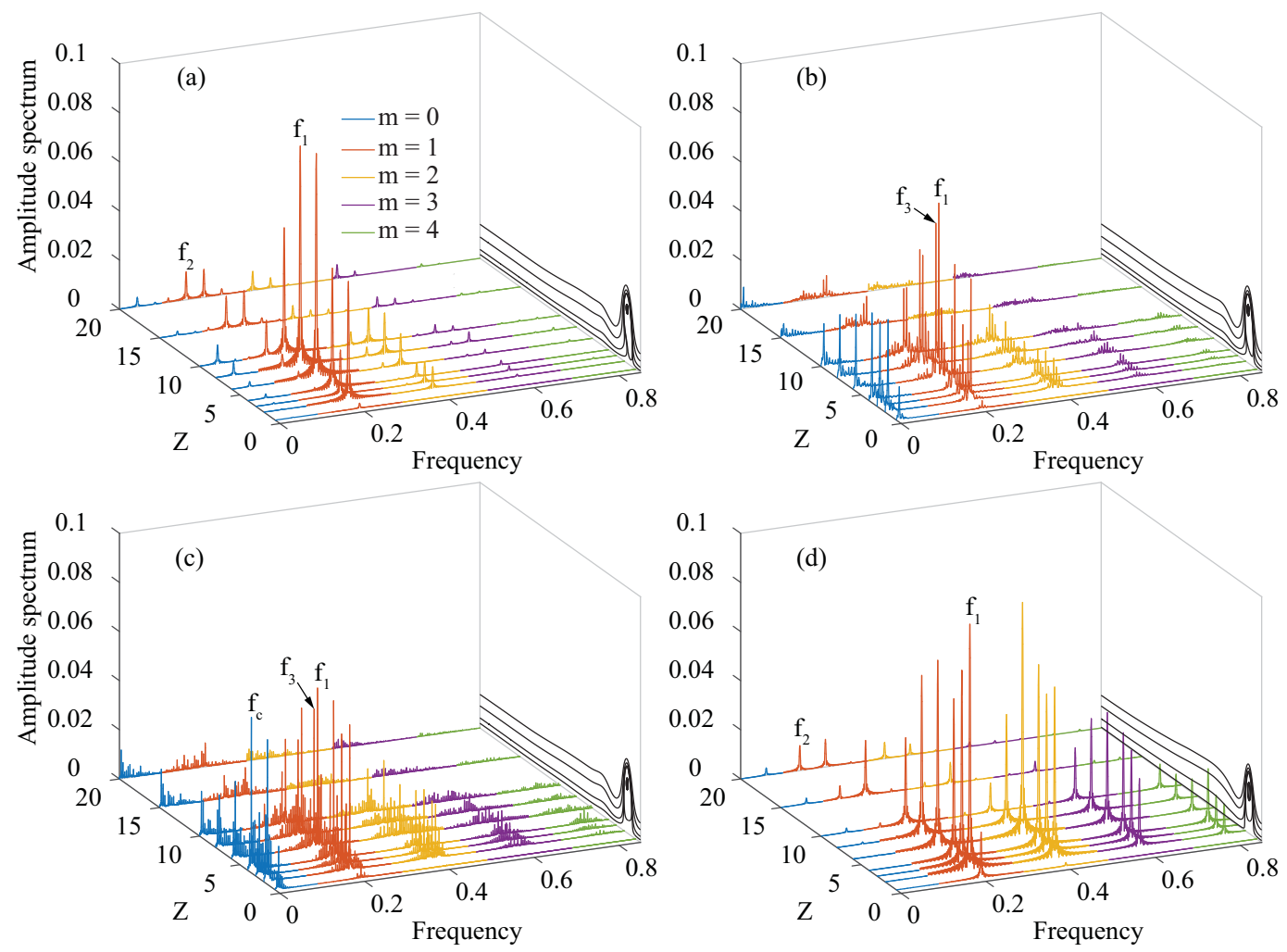

FIG. 12. Temporal-azimuthal Fourier decomposition of the DNS solutions for the Reynolds values (a) $\operatorname{Re}=200$, (b) $\operatorname{Re}=220$, (c) $\operatorname{Re}=230$, and (d) $\operatorname{Re}=250$. Amplitude Fourier spectrum of the tangential velocity $V_{\theta}$ for $R=0.6$ along the $Z$ location $(Z=0.6,2,3,4,6,8,10,15,20)$ with the first five azimuthal wave number $m$ plotted in parallel with the streamlines of the time-average flow.

The chaotic attractor at $\mathrm{Re}=215$ and $\mathrm{Re}=220$ has a third incommensurable frequency that was not found to be associated with any of the available eigenmodes since their spatial locations and/or frequency predictions could not be correlated with DNS results. This questions the ability of global linear analysis to predict the different modes underlying the chaos prevailing in this parameter range.

\section{B. Temporal-azimuthal Fourier series cascade}

The description of the flow patterns appears as incomplete using global linear stability analysis and a temporal-azimuthal Fourier series decomposition of the DNS is therefore performed to further extract spatially developing modes. This decomposition is illustrated in Fig. 12 for the selected Reynolds values $\operatorname{Re}=[200,220,230,250]$. Figure 12 displays the amplitude Fourier spectrum of the tangential velocity $V_{\theta}$ for the radial value $R=0.6$ along several $Z$ stations for the first five azimuthal modes. These spectra are plotted in parallel with the streamlines of the time-average flow to visualize the recirculation bubble position. The azimuthal modes are separated by a frequency range, i.e., the low frequencies represent the azimuthal modes $m=0$, the frequencies around $f \approx 0.2$ represent the azimuthal modes $m=1$, the frequencies around $f \approx 0.4$ represent the azimuthal modes $m=2$, etc.

In all the illustrated cases, strong $m=1$ oscillations are observed up to $Z=10$, meaning that not only the primary spiral spins temporally but also the recirculation bubble. From $Z=10$, the 

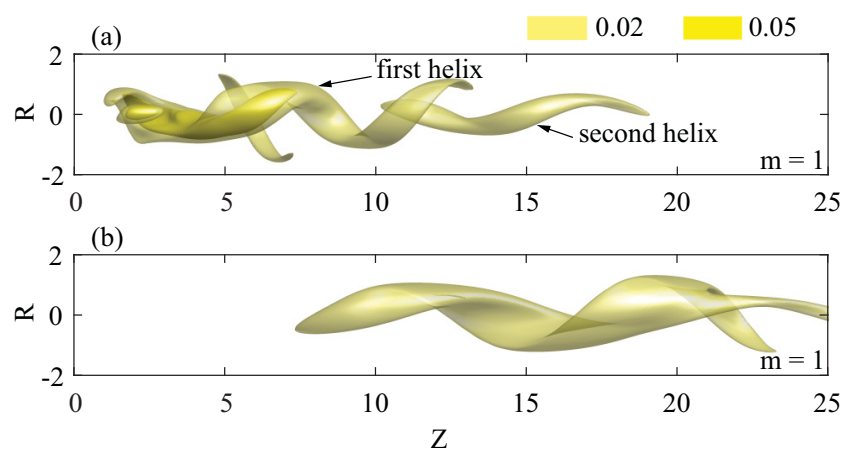

FIG. 13. Axial velocity isocontour of temporal-azimuthal Fourier series modes at $\operatorname{Re}=200$, associated with (a) the primary spiral at frequency $f_{1}$ and (b) the downstream spiral at frequency $f_{2}$ at an arbitrary selected phase.

amplitude of these $m=1$ modes suddenly drops until the end of the domain. This behavior can be correlated to the first and second helices appearing in the primary eigenmode [Fig. 11(a)] where the intensity of the first helix is higher than that of the second helix. In addition, it corresponds to the same $Z$ location, around $Z=10$. These two helices composing the primary spiral will be observed also for Reynolds number values of $\operatorname{Re}=[220,230,250]$ (see Figs. 13-16), as the temporal-azimuthal Fourier series cascade suggests (Fig. 12).

At $\operatorname{Re}=200$ [Fig. 12(a)], the peaks associated with the primary spiral $f_{1}$ and the downstream spiral $f_{2}$ are well defined. These two modes interact through the quadratic nonlinearity of the Navier-Stokes equations and produce an axisymmetric pulsation $m=0$ (labeled $f_{b}$ in Fig. 8) as observed by Ruith et al. [7] at higher swirl number. This axisymmetric mode has its highest amplitude at $Z=10$ where the first helix of the primary spiral [Fig. 11(a)] and the downstream spiral overlap [Fig. 11(b)]. This peak starts around $Z=3$, meaning that the downstream spiral impacts the system until the near wake of the bubble. Moreover, the quasiperiodicity of the system encompasses the full streamwise length.

The chaotic attractor on which the system is folded at $\mathrm{Re}=220$ has three incommensurable frequencies displayed in Fig. 12(b). The main $m=1$ frequency represents the primary spiral, while the downstream spiral associated with $f_{2}$ has a smaller amplitude than at $\operatorname{Re}=200$, although it remains present. In addition, the third incommensurable frequency $f_{3}$, which corresponds to the closest peak of the frequency $f_{1}$ [Fig. 8(i)], is mainly active in the same $Z$ range as the primary spiral, i.e., between $Z=0$ and $Z=10$, and therefore supports the vortical structure development identified as the near-wake spiral in the DNS (Fig. 2). The axisymmetric oscillation of the flow has a larger amplitude than at $\mathrm{Re}=200$ and its frequency corresponds to the difference of the primary and near-wake spiral frequency $f_{c}=f_{1}-f_{3}$. Moreover, this chaotic regime is kept along an extended $Z$ region, which can be viewed by dense attractor cross sections at different $Z$ stations (not shown here).

At $\mathrm{Re}=230$, a quasiperiodic regime on a torus $\mathbb{T}_{2}$ shows several $m=1$ frequencies but the dynamics is governed by the primary $f_{1}$ and the near-wake $f_{3}$ spirals, as observed in Fig. 8(k). Note that the axisymmetric oscillations $m=0$ have a comparable amplitude in the near wake of the recirculation bubble than the oscillations of the azimuthal modes $m=1$ [Fig. 12(c)]. This highlights a strong nonlinear interaction of the $m=1$ modes. The torus $\mathbb{T}_{2}$ at this Reynolds value starts to break down because the downstream spiral starts to be more active from $Z=10 \mathrm{on}$, while, in addition, the amplitude of the primary spiral drops.

The synchronization regime at a Reynolds value of $\mathrm{Re}=250$ is made possible by the frequency match of the primary $f_{1}$ and near-wake $f_{3}$ spiral. This synchronization regime occurs only at $Z$ stations upstream of $Z=10$, as highlighted by a unique peak at the given frequency [Fig. 12(d)]. Downstream of $Z=10$, the downstream spiral emerges, which turns the limit cycle into a torus $\mathbb{T}_{2}$. In 

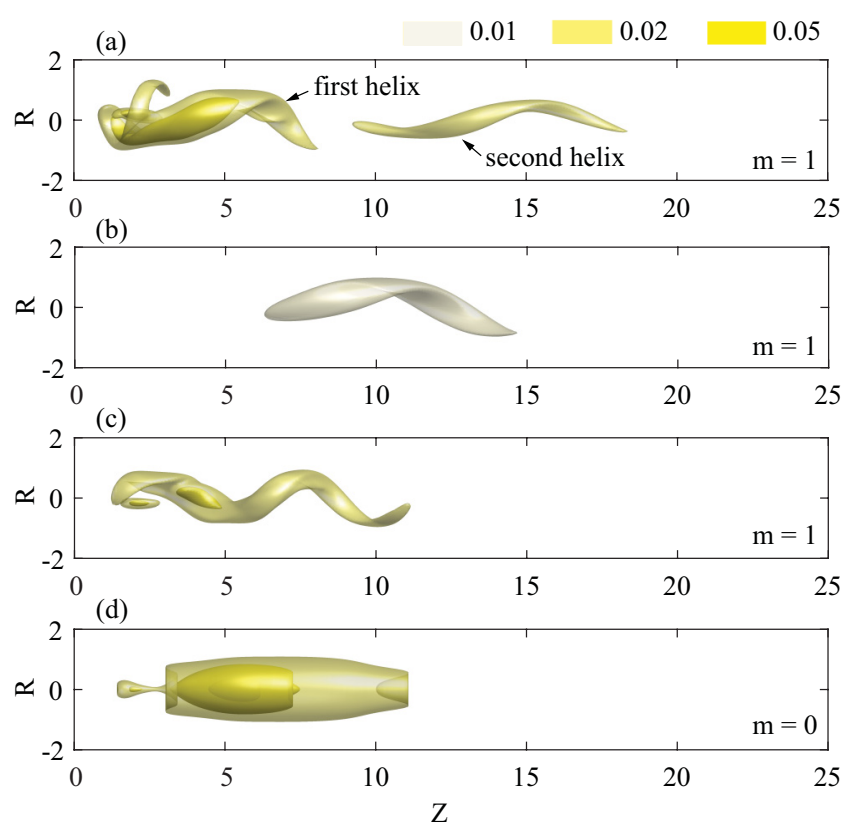

FIG. 14. Axial velocity isocontour of temporal-azimuthal Fourier series modes at $\mathrm{Re}=220$, associated with (a) the primary spiral at frequency $f_{1}$, (b) the downstream spiral at frequency $f_{2}$, (c) the near-wake spiral at frequency $f_{3}$, and (d) the axisymmetric pulsation at frequency $f_{c}$ at an arbitrary selected phase.

addition, the synchronized region has a first harmonic frequency $f_{a} \simeq 0.4$ of amplitude comparable to that of the fundamental frequency $f_{1}$, which suggests that its associated double-helix superharmonic mode can be involved in the nonlinear dynamics [Fig. 8(n)].

\section{Temporal-azimuthal Fourier series modes}

The modes of the temporal-azimuthal Fourier series decomposition, extracted using frequency selection, are displayed as axial velocity isocontours to provide an overview of their structures in Figs. 13-16. The juxtaposition of these structures with the cascade of the amplitude Fourier spectra (Fig. 12), the global instability properties (Fig. 10), and the vortical structures identified in the DNS (Fig. 8) enables the identification of the patterns, which contribute to the dynamics of this swirling flow. As previously demonstrated by the global stability analysis, the frequencies $f_{1}$ and $f_{2}$ correspond to the primary and downstream spirals for all considered Reynolds values. Moreover, the axial velocity isocontours of this Fourier series decomposition at a Reynolds value of $\mathrm{Re}=200$ [see Figs. 13(a) and 13(b)] appear similar to the respective eigenmodes obtained by the global linear stability analysis (Fig. 11), while a different normalization is considered due to undefined magnitude of eigenmodes. The primary spiral has two single helices [Fig. 13(a)]: the first helix at the beginning of the computational domain and the second helix downstream. This first helix develops on the recirculation bubble and extends in its wake, which already emphasizes the oscillation of the bubble followed by a single spiral [see Fig. 8(d)]. The downstream spiral [Fig. 13(b)] has only a single helix, which overlaps with both helices of the primary spiral.

The consistency of both methods at $\mathrm{Re}=200$ leads us to investigate by Fourier series decomposition the third incommensurable frequency $f_{3}$ (the near-wake spiral), which turns the quasiperiodicity into a chaotic regime in the range of Reynolds values $\mathrm{Re}=215-220$, while global stability analysis fails to identify this mode. Figure 14 displays the primary, downstream, near-wake 

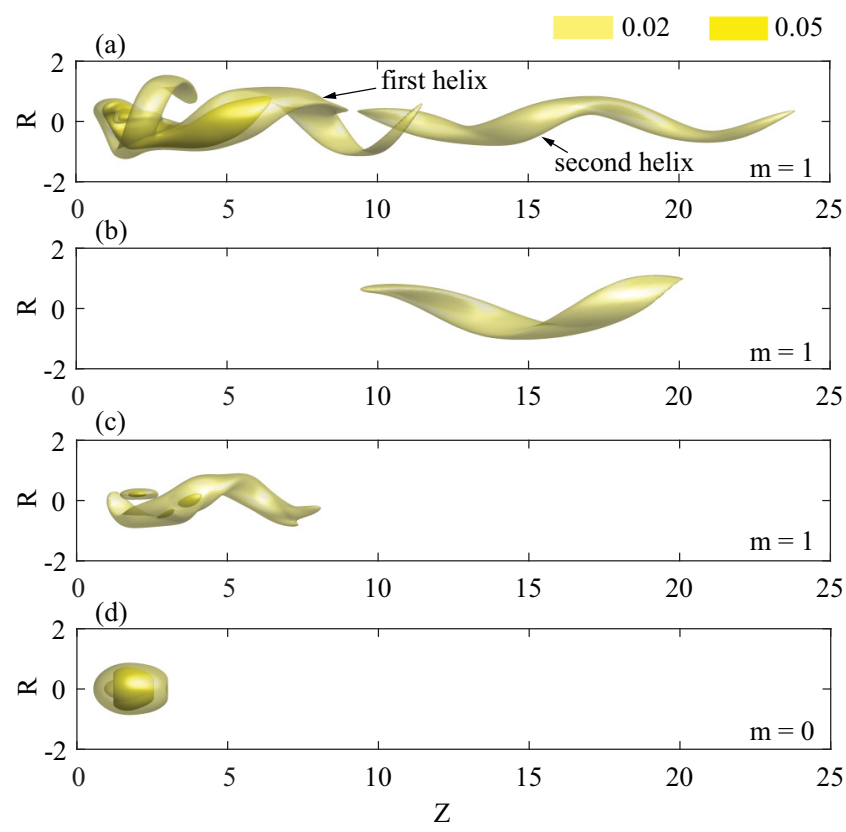

FIG. 15. Axial velocity isocontour of temporal-azimuthal Fourier series modes at $R e=230$, associated with (a) the primary spiral at frequency $f_{1}$, (b) the downstream spiral at frequency $f_{2}$, (c) the near-wake spiral at frequency $f_{3}$, and (d) the axisymmetric pulsation at frequency $f_{c}$ at an arbitrary selected phase.

spiral, and axisymmetric modes extracted for the frequencies $f_{1}, f_{2}, f_{3}$, and $f_{c}$, respectively. The structure of the primary spiral is conserved, the first single helix developing on the recirculation bubble and extending in its wake as well as a second helix emerging downstream [Fig. 14(a)]. The downstream spiral, which has a smaller amplitude, arises in the middle of the two helices of the primary spiral [Fig. 14(b)]. The near-wake spiral develops also on the recirculation bubble and its near wake and overlaps with both primary and downstream spirals [Fig. 14(c)]. The axisymmetric mode resulting from the nonlinear interactions of the primary and near-wake spirals influences a large zone of the flow up to $Z=11$ [Fig. 14(d)].

At a Reynolds value of $\mathrm{Re}=230$, a $\mathbb{T}_{2}$ torus is observed [Fig. 7(f)] and the Fourier modes associated with this quasiperiodic regime are displayed in Fig. 15. The axial velocity isocontours display the same patterns as for the previous Reynolds values $(\operatorname{Re}=220)$ : two dissociated helices for the primary spiral [Fig. 15(a)], a single downstream helix for the downstream spiral [Fig. 15(b)] and a single helix in the near wake of the recirculation bubble for the near-wake spiral [Fig. 15(c)]. In contrast, the axisymmetric mode [Fig. 15(d)] is focused on the recirculation bubble, which suggests a better phase correlation of the different single-helical modes and yielding the strategic repositioning of the frequencies forming this torus $\mathbb{T}_{2}$ at $\operatorname{Re}=230$.

The axial velocity isocontours of the Fourier series decomposition for the synchronized regime at a Reynolds value of $\operatorname{Re}=250$ are displayed in Fig. 16. The primary spiral [Fig. 16(a)], which in this state embeds the near-wake spiral $f_{3}$, shows a complex structure with several single helices. The strongest helix at the beginning of the domain extends and overlaps with the second downstream helix, in contrast to $\operatorname{Re}=230$ and $\operatorname{Re}=220$, where these helices are spatially shifted. The downstream spiral migrates further downstream [Fig. 16(b)], as observed in Fig. 12(d), which is likely to result from the frequency synchronization regime at the beginning of the domain.

Since the first harmonic of the primary spiral (labeled $f_{a}$ ) has an amplitude comparable to that of the fundamental frequency $f_{1}$ in the cascade of the amplitude Fourier spectra [Fig. 12(d)], the mode associated with this harmonic, called a double-helix superharmonic, is displayed in Fig. 16(c). This 

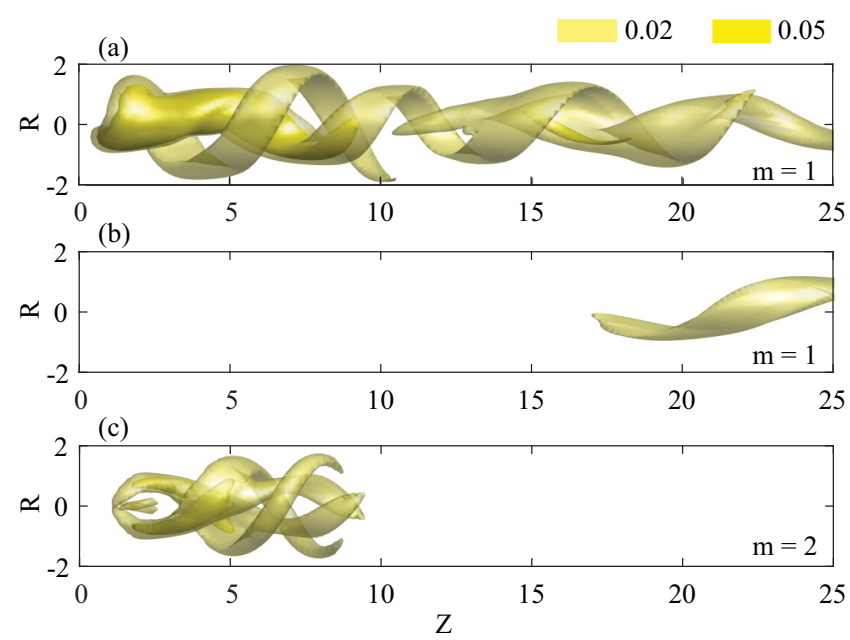

FIG. 16. Axial velocity isocontour of temporal-azimuthal Fourier series modes at $\mathrm{Re}=250$, associated with (a) the primary spiral at frequency $f_{1}$, (b) the downstream spiral at frequency $f_{2}$, and (c) the double-helix first harmonic (labeled $f_{a}$ ) at an arbitrary selected phase.

double helix appears at the beginning of the domain and could therefore contribute to the nonlinear dynamics to form the two vortical structures observed in Fig. 8(n).

\section{CONCLUSION}

The nonlinear dynamics of the unconfined open swirling flow stemming from a GrabowskiBerger [29] vortex is studied for a fixed swirl number of $S=1.095$ and a Reynolds number up to $\mathrm{Re}=300$. As the Reynolds number increases, this flow reveals a Ruelle-Takens-Newhouse route to chaos establishing a chaotic regime between the Reynolds values of $\operatorname{Re}=215$ and $\operatorname{Re}=220$. A first supercritical Hopf bifurcation, leading to the spiral vortex breakdown [7,8], is encountered at the instability threshold of $\operatorname{Re}=143.5[14,35]$, yielding the so-called primary spiral in the present study. A subsequent supercritical Hopf bifurcation turns the flow into a quasiperiodic regime on a torus $\mathbb{T}_{2}$ at $\operatorname{Re}=200$ as shown by a global stability analysis around the base flow in Ref. [35]. Then a quasiperiodic regime with three incommensurable frequencies is identified at a Reynolds value of $\operatorname{Re}=205$. Sensitivity to the initial condition suggests that the present attractor is a stable torus $\mathbb{T}_{3}$, similar to that in [46]. The interpretation of this regime as a stable torus $\mathbb{T}_{3}$ is not in contradiction with the Ruelle-Takens-Newhouse route to chaos since the latter does not exclude such nonchaotic states [47].

Frequency identification based on the amplitude Fourier spectra shows three incommensurable frequencies associated with single-helical spirals. The first $f_{1}$ and the third $f_{3}$ have a peak of amplitude close to each other, while the second $f_{2}$ frequency has a peak of amplitude which remains at a lower frequency. As the Reynolds number is increased further, these three peaks become closer, leading to a stronger axisymmetric mode at low frequency (labeled $f_{c}$ in Fig. 8) generated by the nonlinear interactions. Hence, the potentially stable torus $\mathbb{T}_{3}$ observed at $\operatorname{Re}=205$ breaks down, yielding a chaotic regime at Reynolds values from $\operatorname{Re}=215$ to $\mathrm{Re}=220$.

Since an unconfined geometry is considered, a quasiperiodic or chaotic regime should be generated by nonlinear interactions of pure hydrodynamic instabilities. In fact, the first bifurcation of the flow associated with the spiral vortex breakdown is a self-sustained instability $[8,11,14]$. Local, weakly nonparallel stability analysis and global stability analysis around a fixed-point solution successfully predict this instability threshold, while the prediction from the linearization around the base flow 
quickly fails to predict the limit cycle frequency as the Reynolds number is increased [35]. Global stability analysis around the mean flow, in contrast, successfully predicts the frequency of the spiral vortex breakdown and identifies two of the three incommensurable frequencies as the single-helical spiral of azimuthal wave number $m=1$. The first frequency $f_{1}$ is an unstable eigenmode, the primary spiral, which has two spatially shifted single helices, the first helix on the recirculation bubble and in its near wake and the second downstream helix. The second frequency $f_{2}$ is associated with a linearly stable eigenmode, called the downstream spiral, which solely develops in the wake of the bubble. Hence, an excitation mechanism induced by the advection of fluctuations should occur to lead the downstream spiral to develop its own oscillation.

In addition, the third incommensurable frequency $f_{3}$, which is definitely needed to observe a chaotic regime, fails to be identified as an eigenmode from the global stability analysis. Moreover, axisymmetric $m=0$ or double-helical $m=2$ modes cannot be associated with corresponding frequency or velocity distributions. A temporal-azimuthal Fourier series decomposition is therefore performed and allows us to extract the flow pattern of these incommensurable frequencies and to corroborate also the previously identified eigenmodes. It appears that $f_{3}$ is a single-helical mode developing in the near wake of the recirculation bubble, which overlaps with the primary spiral. The successive emergence and the nonlinear interactions of the primary, downstream, and near-wake single spirals, also observed as a vortical structure in the DNS results, yield a Ruelle-Takens-Newhouse route to chaos.

A torus $\mathbb{T}_{2}$ with two incommensurable frequencies is observed at a larger Reynolds value of $\operatorname{Re}=230$, while three single spirals are identified at the previous Reynolds value of $\operatorname{Re}=220$. The following necessary frequency and mode reassortment are observed. The downstream spiral frequency becomes a subharmonic of those two incommensurable frequencies and a fourth frequency is, in addition, identified as commensurable with the two driving frequencies. This attractor is ultimately characterized by two incommensurable frequencies, which are associated with the primary and near-wake spirals. The spatial location of these spirals appears, as a consequence, highly correlated to enable this torus $\mathbb{T}_{2}$, while at the chaotic regime $\mathrm{Re}=220$ these vortical structures are largely uncorrelated.

At a Reynolds value of $\mathrm{Re}=250$ a synchronized regime appears, formally identified in the amplitude Fourier spectrum cascade. We could not determine if this results or originates from the migration of the downstream spiral associated with the frequency $f_{2}$ further downstream. Therefore, a limit cycle is observed at the beginning of the domain by frequency synchronization of the primary and near-wake spiral, which turns into a torus $\mathbb{T}_{2}$ downstream of $Z=10$. Vorticity isosurfaces of the DNS results exhibit two spirals at $\mathrm{Re}=250$ [Fig. 8(n)], which contrast with the single peak of the amplitude Fourier spectrum [Fig. 8(m)]. We suggest that these nonlinear structures are a suitable phase and frequency combination of the two helices of the primary spiral and its double-helical superharmonic mode, associated with the frequency $f_{a}=2 f_{1}$. Actually, the amplitude of the first harmonic of the primary spiral $a$ is comparable to that of its fundamental $f_{1}$ [see Fig. 12(d)] and the double-helix first harmonic overlaps with the first helix of the primary mode at the beginning of the domain [see Fig. 16(c)]. Although this double-helix first harmonic appears to contribute to the flow dynamics, the origin of this synchronized state is the development of two single-helical modes as demonstrated by the global stability analysis (see Fig. 10), as for the other Reynolds values.

Finally, after this synchronization regime, the flow turns into a subsequent chaotic regime at $\operatorname{Re} \approx 300$ with three incommensurable frequencies, which is suspected to definitely hold for larger Reynolds numbers, where the turbulence transition will be further exacerbated.

Self-sustained instabilities are able to generate a chaotic regime in this open flow starting from the globally unstable region before turning into turbulence at higher Reynolds number. A stable torus $\mathbb{T}_{3}$ is furthermore observed in a narrow low-Reynolds-number band around $\operatorname{Re}=210$. 

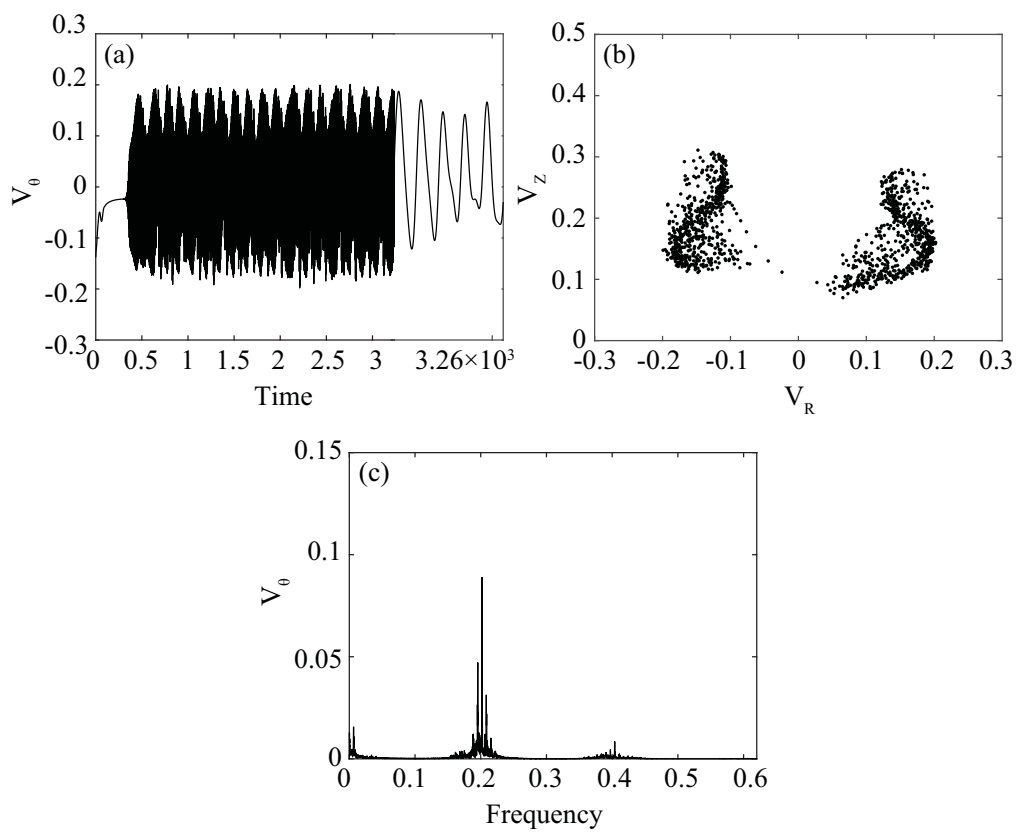

FIG. 17. (a) Time series, (b) attractor cross section, and (c) amplitude Fourier spectrum at the monitoring point $(R, \theta, Z)=(0.1,0.0,6.0)$ and at a Reynolds value of $\mathrm{Re}=220$ computed on the finer discretization M3.

\section{ACKNOWLEDGMENTS}

The authors are thankful to the Swiss National Science Foundation (Grant No. 200021 149818.1), which has supported this work. The authors are also grateful to T. Schneider and P. Manneville, who provided insight and expertise that greatly assisted the research.

\section{APPENDIX A: THREE-DIMENSIONAL DNS VALIDATION}

The 3D direct numerical flow simulations are validated by changing the mesh size, the mesh topology, the boundaries, the domain dimensions, and the time step for a fixed swirl number $S=$ 1.095 and Reynolds number $\mathrm{Re}=200$. The convergence criterion is based on the deviation of the frequency and the amplitude of the radial velocity component of the single-helical vortex flow. These values are monitored at $(R, \theta, Z)=(0.1,0.0,5.0)$, which is located downstream the bubble, in the helix. The results of this convergence study are displayed in Table II. The topology of the mesh is an O-grid type with two different cells arrangements in the center: diamonds cells that almost preserve the azimuthal symmetry of the problem and square cells. All cells have $P_{10}$ and $P_{8}$ spectral discretizations with uniform numbers of nodes $n_{x} \times n_{y} \times n_{z}=10 \times 10 \times 10$ and $n_{x} \times n_{y} \times n_{z}=$

TABLE II. Convergence of the 3D numerical flow simulations and eigenvalues of the mean flow.

\begin{tabular}{llccccccc}
\hline \hline Case & Topology & BC on $\Gamma_{\text {ext }}$ & $L_{z}$ & $L_{r}$ & $n_{e}$ & CFL & 3D (amplitude,frequency) & Eigenvalue \\
\hline M1 & Diamonds & Free outflow & 40 & 50 & 11040 & 0.37 & $(0.1299,0.1991)$ & $(0.01427,1.25009)$ \\
M2 & Diamonds & Free outflow & 60 & 50 & 16320 & 0.37 & $(0.1224,0.1991)$ & $(0.01454,1.25193)$ \\
M3 & Diamonds & Free outflow & 40 & 50 & 29696 & 0.44 & $(0.1284,0.1984)$ & $(0.01475,1.25175)$ \\
M4 & Squares & No slip & 40 & 50 & 12512 & 0.34 & $(0.1231,0.1991)$ & \\
M5 & Diamonds & Free outflow & 40 & 50 & 11040 & 0.18 & $(0.1267,0.1991)$ & \\
\hline \hline
\end{tabular}


TABLE III. Frequency interaction for $\operatorname{Re}=230, S=1.095$ at $R=0.2$, and $Z=6.0$ measured and approximated by the formulas using $f_{1}, f_{2}, f_{3}$, and $f_{4}$.

\begin{tabular}{lcc}
\hline \hline Frequency & Harmonics & DNS results \\
\hline$f_{1}$ & $f_{1}$ & 0.202274 \\
$f_{2}$ & $6 f_{1}-5 f_{3}=0.152492$ & 0.152683 \\
$f_{3}$ & $f_{3}$ & 0.193977 \\
$f_{4}$ & $10 f_{3}-6 f_{1}=0.181531$ & 0.181579 \\
$f_{a}$ & $2 f_{1}=0.40454$ & 0.40454 \\
$f_{b}$ & $5 f_{3}-5 f_{1}=0.049782$ & \\
$f_{c}$ & $f_{1}-f_{3}=0.008297$ & 0.008296 \\
$f_{d}$ & $2 f_{1}-f_{3}=0.210571$ & 0.210571 \\
$f_{e}$ & $2 f_{3}-f_{1}=0.18568$ & 0.185775 \\
$f_{h}$ & $11 f_{3}-7 f_{1}=0.173282$ & 0.173377 \\
$f_{i}$ & $9 f_{3}-5 f_{1}=0.189876$ & 0.189876 \\
\hline \hline
\end{tabular}

$8 \times 8 \times 8$ for velocities and pressure, respectively. The robustness of the solutions in front of the convective boundary condition on the outlet $\Gamma_{\text {out }}$ is investigated by changing the length of the domain to $Z_{\max }=60$ for mesh M2 instead of $Z_{\max }=40$. The radial unconfinement is guaranteed by the invariance of the external boundary conditions. Two different types were investigated: a free-stress boundary and a no-slip boundary, mesh M4. The CFL number invariance is investigated on mesh M5 and a refined mesh M3 concludes the convergence analysis. Mesh M1 is the reference mesh for this study.

The time series, the attractor cross section, and the Fourier amplitude spectrum of the chaotic regime identified at a Reynolds value of $\mathrm{Re}=220$ are displayed in Fig. 17 for the finer discretization M3. The irregular fluctuations of the time series are again observed for this computation.

\section{APPENDIX B: VALIDATION OF THE FREQUENCY ATTRIBUTIONS}

The frequency measured on the Fourier amplitude spectrum and the approximation based on the mean frequency are reported in Table III. Excellent correspondence is observed. Note that the $b$ frequency, resulting from the nonlinear interaction of the primary and the downstream spiral (at $f_{1}$ and $f_{2}$, respectively), is not observed at this monitoring point for this value of Reynolds.

[1] O. Lucca-Negro and T. O'Doherty, Vortex breakdown: A review, Prog. Energy Combust. Sci. 27, 431 (2001).

[2] I. Gursul, Z. Wang, and E. Vardaki, Review of flow control mechanisms of leading-edge vortices, Prog. Aerosp. Sci. 43, 246 (2007).

[3] S. Pasche, F. Avellan, and F. Gallaire, Part load vortex rope as a global unstable mode, J. Fluids Eng. 139, 051102 (2017).

[4] C. O. Paschereit, P. Flohr, and E. J. Gutmark, Combustion control by vortex breakdown stabilization, J. Turbomach. 128, 679 (2002).

[5] P. Huerre and P. A. Monkewitz, Local and global instabilities in spatially developing flows, Annu. Rev. Fluid Mech. 22, 473 (1990).

[6] I. Delbende, J.-C. Chomaz, and P. Huerre, Absolute/convective instabilities in the Batchelor vortex: A numerical study of the linear impulse response, J. Fluid Mech. 355, 229 (1998). 
[7] M. R. Ruith, P. Chen, E. Meiburg, and T. Maxworthy, Three-dimensional vortex breakdown in swirling jets and wakes: Direct numerical simulation, J. Fluid Mech. 486, 331 (2003).

[8] F. Gallaire, M. Ruith, E. Meiburg, J.-M. Chomaz, and P. Huerre, Spiral vortex breakdown as a global mode, J. Fluid Mech. 549, 71 (2006).

[9] V. Theofilis, Global linear instability, Annu. Rev. Mech. 43, 319 (2011).

[10] P. Meliga, G. Pujals, and E. Serre, Sensitivity of 2-D turbulent flow past a D-shaped cylinder using global stability, Phys. Fluids 24, 061701 (2012).

[11] U. A. Qadri, D. Mistry, and M. P. Juniper, Structural sensitivity of spiral vortex breakdown, J. Fluid Mech. 720, 558 (2013).

[12] E. Vyazmina, J. W. Nichols, J.-M. Chomaz, and P. J. Schmid, The bifurcation structure of viscous steady axisymmetric vortex breakdown with open lateral boundaries, Phys. Fluids 21, 074107 (2009).

[13] Z. Rusak, J. Granata, and S. Wang, An active feedback flow control theory of the axisymmetric vortex breakdown process, J. Fluid Mech. 774, 488 (2015).

[14] P. Meliga, F. Gallaire, and J.-M. Chomaz, A weakly nonlinear mechanism for mode selection in swirling jets, J. Fluid Mech. 699, 216 (2012).

[15] H. B. Squire, Analysis of the vortex breakdown phenomenon, Part I, Aero. Dept., Imperial College of Science and Technology, Rep. no. 102.

[16] J. M. Lopez, On the bifurcation structure of axisymmetric vortex breakdown in a constricted pipe, Phys. Fluids 6, 3683 (1994).

[17] M. P. Escudier and N. Zehnder, Vortex-flow regimes, J. Fluid Mech. 115, 105 (1982).

[18] P. Wulf, C. Egbers, and H. J. Rath, Routes to chaos in wide-gap spherical Couette flow, Phys. Fluids 11, 1359 (1999).

[19] S. W. Morris, E. Bodenschatz, D. S. Cannell, and G. Ahlers, Spiral Defect Chaos in Large Aspect Ratio Rayleigh-Bénard Convection, Phys. Rev. Lett. 71, 2026 (1993).

[20] D. A. Egolf, I. V. Melnikov, W. Pesch, and R. E. Ecke, Mechanisms of extensive spatiotemporal chaos in Rayleigh-Bénard convection, Nature (London) 404, 733 (2000).

[21] F. Sotiropoulos, Y. Ventikos, and T. C. Lackey, Chaotic advection in three-dimensional stationary vortexbreakdown bubbles: Sil'nikov's chaos and the devil's staircase, J. Fluid Mech. 444, 257 (2001).

[22] E. Serre and P. Bontoux, Vortex breakdown in a three-dimensional swirling flow, J. Fluid Mech. 459, 347 (2002).

[23] J. M. Lopez, Rotating and modulated rotating waves in transitions of an enclosed swirling flow, J. Fluid Mech. 553, 323 (2006).

[24] J. N. Sørensen and E. A. Christensen, Direct numerical simulation of rotating fluid flow in a closed cylinder, Phys. Fluids 7, 764 (1995).

[25] C. H. Amon, A. M. Guzmán, and B. Morel, Lagrangian chaos, Eulerian chaos, and mixing enhancement in converging diverging channel flows, Phys. Fluids 8, 1192 (1996).

[26] S. Newhouse, D. Ruelle, and F. Takens, Occurrence of strange axiom A attractors near quasi periodic flows on $T^{m}, m \geqq 3$, Commun. Math. Phys. 64, 35 (1978).

[27] M. J. Feigenbaum, The metric universal properties of period doubling bifurcations and the spectrum for a route to turbulence, Ann. N.Y. Acad. Sci. 357, 330 (1980).

[28] Y. Pomeau and P. Manneville, Intermittent transition to turbulence in dissipative dynamical systems, Commun. Math. Phys. 74, 189 (1980).

[29] W. J. Grabowski and S. A. Berger, Solutions of the Navier-Stokes equations for vortex breakdown, J. Fluid Mech. 75, 525 (1976).

[30] P. F. Fischer, J. W. Lottes, and S. G. Kerkemeier, NEK5000, 2008, available at http://nek5000.mcs.anl.gov.

[31] M. Frigo and S. G. Johnson, The design and implementation of FFTW3, Proc. IEEE 93, 216 (2005), special issue on program generation, optimization, and platform adaptation.

[32] D. Barkley, Linear analysis of the cylinder wake mean flow, Europhys. Lett. 75, 750 (2006).

[33] S. E. Turton, L. S. Tuckerman, and D. Barkley, Prediction of frequencies in thermosolutal convection from mean flows, Phys. Rev. E 91, 043009 (2015).

[34] O. Tammisola and M. P. Juniper, Coherent structures in a swirl injector at $\operatorname{Re}=4800$ by nonlinear simulations and linear global modes, J. Fluid Mech. 792, 620 (2016). 
[35] S. Pasche, F. Gallaire, and F. Avellan, Predictive control of spiral vortex breakdown, J. Fluid Mech. 842, 58 (2018).

[36] V. Mantič-Lugo, C. Arratia, and F. Gallaire, Self-Consistent Mean Flow Description of the Nonlinear Saturation of the Vortex Shedding in the Cylinder Wake, Phys. Rev. Lett. 113, 084501 (2014).

[37] D. Sipp and A. Lebedev, Global stability of base and mean flows: A general approach and its applications to cylinder and open cavity flows, J. Fluid Mech. 593, 333 (2007).

[38] P. Meliga, Harmonics generation and the mechanics of saturation in flow over an open cavity: A secondorder self-consistent description, J. Fluid Mech. 826, 503 (2017).

[39] M. R. Khorrami, A Chebyshev spectral collocation method using a staggered grid for the stability of cylindrical flows, Int. J. Numer. Methods Fluids 12, 825 (1991).

[40] F. Hecht, New development in freefem++, J. Numer. Math. 20, 251 (2012).

[41] R. B. Lehoucq, D. C. Sørensen, and C. Yang, ARPACK Users' Guide (SIAM, Philadelphia, 1998), available at http://www.caam.rice.edu/software/ARPACK/.

[42] J. C. Sprott, Elegant Chaos: Algebraically Simple Chaotic Flows (World Scientific, Singapore, 2010).

[43] See Supplemental Material at http://link.aps.org/supplemental/10.1103/PhysRevFluids.3.064701 for movies.

[44] J. P. Gollub and S. V. Benson, Many routes to turbulent convection, J. Fluid Mech. 100, 449 (1980).

[45] K. Kashinath, I. C. Waugh, and M. P. Juniper, Nonlinear self-excited thermoacoustic oscillations of a ducted premixed flame: Bifurcations and routes to chaos, J. Fluid Mech. 761, 399 (2014).

[46] L. Oteski, Y. Duguet, L. Pastur, and P. Le Quéré, Quasiperiodic routes to chaos in confined two-dimensional differential convection, Phys. Rev. E 92, 043020 (2015).

[47] J.-P. Eckmann, Roads to turbulence in dissipative dynamical systems, Rev. Mod. Phys. 53, 643 (1981).

[48] C. Grebogi, E. Ott, and J. A. Yorke, Are Three-Frequency Quasiperiodic Orbits to be Expected in Typical Nonlinear Dynamical Systems? Phys. Rev. Lett. 51, 339 (1983).

[49] F. Gallaire and J.-C. Chomaz, Mode selection in swirling jet experiments: A linear stability analysis, J. Fluid Mech. 494, 223 (2003).

[50] O. Marquet, D. Sipp, and L. Jacquin, Sensitivity analysis and passive control of cylinder flow, J. Fluid Mech. 615, 221 (2008).

[51] A. Pimenov, S. Slepneva, G. Huyet, and A. G. Vladimirov, Dispersive Time-Delay Dynamical Systems, Phys. Rev. Lett. 118, 193901 (2017). 